\title{
Faraday rotation measure synthesis ${ }^{\star}$
}

\author{
M. A. Brentjens ${ }^{1,2}$ and A. G. de Bruyn ${ }^{2,1}$ \\ 1 Kapteyn Astronomical Institute, University of Groningen, PO Box 800, 9700 AV Groningen, The Netherlands \\ e-mail: m.a.brentjens@astro.rug.nl \\ 2 ASTRON, PO Box 2, 7990 AA Dwingeloo, The Netherlands
}

Received 4 March 2005 / Accepted 20 June 2005

\section{ABSTRACT}

We extend the rotation measure work of Burn $\left(1966\right.$, MNRAS, 133, 67) to the cases of limited sampling of $\lambda^{2}$ space and non-constant emission spectra. We introduce the rotation measure transfer function (RMTF), which is an excellent predictor of $n \pi$ ambiguity problems with the $\lambda^{2}$ coverage. Rotation measure synthesis can be implemented very efficiently on modern computers. Because the analysis is easily applied to wide fields, one can conduct very fast RM surveys of weak spatially extended sources. Difficult situations, for example multiple sources along the line of sight, are easily detected and transparently handled. Under certain conditions, it is even possible to recover the emission as a function of Faraday depth within a single cloud of ionized gas. Rotation measure synthesis has already been successful in discovering widespread, weak, polarized emission associated with the Perseus cluster (de Bruyn \& Brentjens 2005, A\&A, 441, 931). In simple, high signal to noise situations it is as good as traditional linear fits to $\chi$ versus $\lambda^{2}$ plots. However, when the situation is more complex or very weak polarized emission at high rotation measures is expected, it is the only viable option.

Key words. methods: data analysis - techniques: polarimetric - magnetic fields - polarization - ISM: magnetic fields Cosmology: large-scale structure of Universe

\section{Introduction}

Polarization observations at radio frequencies are an important diagnostic tool in the study of galactic and extragalactic magnetic fields (e.g. Kronberg 1994; Vallee 1997; Widrow 2002). Due to birefringence of the magneto-ionic medium, the polarization angle of linearly polarized radiation that propagates through the plasma is rotated as a function of frequency. This effect is called Faraday rotation. There exist many papers describing aspects of Faraday rotation work. The most relevant ones for this work are Burn (1966), Gardner \& Whiteoak (1966), Sokoloff et al. (1998), Sokoloff et al. (1999), and Vallee (1980).

Assuming that the directions of the velocity vectors of the electrons gyrating in a magnetized plasma are isotropically distributed, Le Roux (1961) showed that the intrinsic degree of polarization of synchrotron radiation from plasma in a uniform magnetic field is given by

$\|p\|=\frac{3 \gamma+3}{3 \gamma+7}$,

independent of frequency and viewing angle. In this equation, $\gamma$ is the spectral index of the relativistic electron distribution in energy

$n_{\mathrm{e}}(E) \mathrm{d} E=A E^{-\gamma} \mathrm{d} E$,

\footnotetext{
* Appendices are only available in electronic form at
} http://www. edpsciences.org where $n_{\mathrm{e}}(E) \mathrm{d} E$ is the density of the electrons between energies $E$ and $E+\mathrm{d} E$. The density of electrons having energies between 1 and $1+\mathrm{d} E$ is $A \mathrm{~d} E$. The total electron density $n_{\mathrm{e}}=\int_{\mathrm{E}_{0}}^{\infty} n_{\mathrm{e}}(E) \mathrm{d} E$, where $E_{0}$ is a cutoff energy that is required in order to let the integral converge.

From observations of the Crab nebula by Woltjer (1958), Westfold (1959) determined that $\gamma \approx \frac{5}{3}$. This would imply a polarization fraction of approximately $67 \%$, independent of frequency. In many radio sources, the observed polarization fractions are much lower. Usually the polarization fraction decreases steeply with increasing wavelength (Conway \& Strom 1985; Strom \& Conway 1985).

Burn (1966) discusses this depolarization effect extensively. One of the mechanisms he discusses is Faraday dispersion: emission at different Faraday depths along the same line of sight.

Following Burn (1966), we make a clear distinction between Faraday depth $(\phi)$ and rotation measure (RM). We define the Faraday depth of a source as

$\phi(\boldsymbol{r})=0.81 \int_{\text {there }}^{\text {here }} n_{\mathrm{e}} \boldsymbol{B} \cdot \mathrm{d} \boldsymbol{r} \mathrm{rad} \mathrm{m}^{-2}$,

where $n_{\mathrm{e}}$ is the electron density in $\mathrm{cm}^{-3}, \boldsymbol{B}$ is the magnetic induction in $\mu$ Gauss, and $\mathrm{d} \boldsymbol{r}$ is an infinitesimal path length in parsecs. A positive Faraday depth implies a magnetic field pointing towards the observer. There may exist many different sources of radiation at different Faraday depths along the 

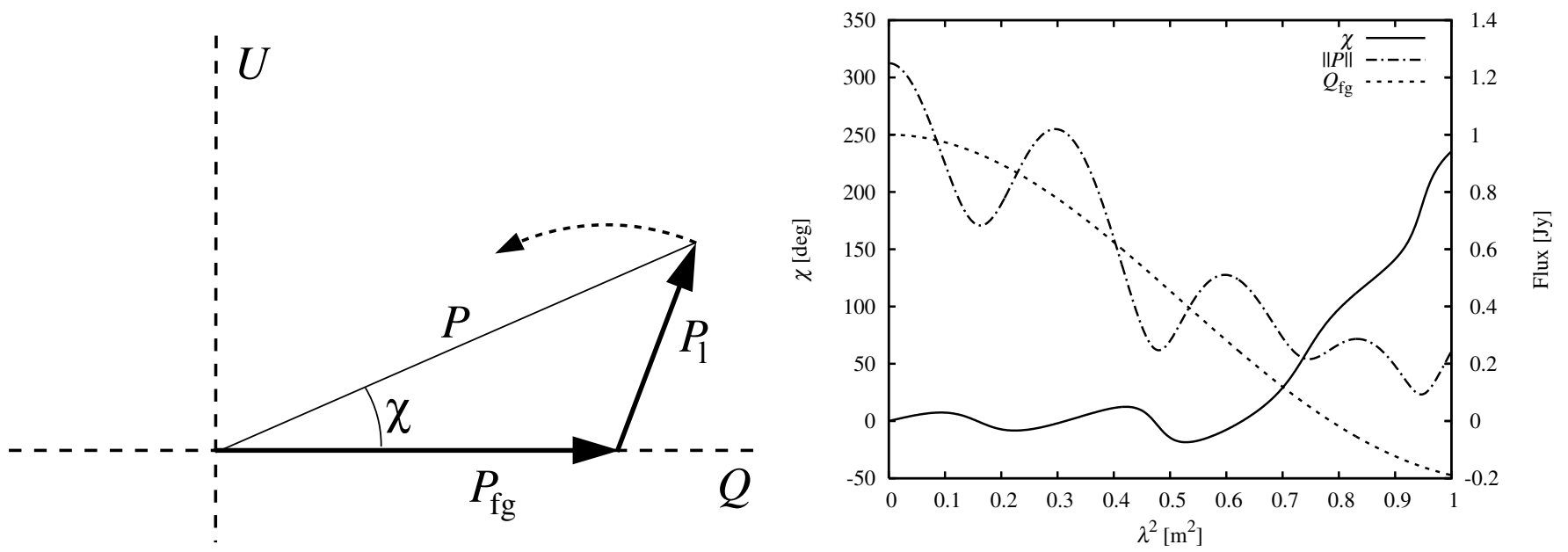

Fig. 1. $P\left(\lambda^{2}\right)$ for a simple, yet non-trivial Faraday dispersion function: a synchrotron emitting and Faraday rotating slab at $-2 \leq \phi \leq+2$ rad $\mathrm{m}^{-2}$ (the Galactic foreground) and a Faraday thin source at $\phi=+10 \mathrm{rad} \mathrm{m}^{-2}$ (a distant radio lobe). The lefthand panel shows qualitatively how the individual $(Q, U)$ vectors of the foreground and the lobe relate to the total polarization $\|P\|$ and the polarization angle $\chi$. It reflects the situation around $\lambda^{2} \approx 0.38 \mathrm{~m}^{2} . P_{1}$ rotates counter clockwise around the moving pivot $P_{\mathrm{fg}}$. The righthand panel shows the polarization angle as a function of $\lambda^{2}$, plotted along the lefthand vertical axis. The total polarization and Stokes $Q$ of the foreground are plotted along the righthand vertical axis.

same line of sight. These sources may be either Faraday thin or Faraday thick. A source is Faraday thin if $\lambda^{2} \Delta \phi \ll 1 . \Delta \phi$ denotes the extent of the source in $\phi$. Faraday thin sources are well approximated by Dirac $\delta$-functions of $\phi$. A source is Faraday thick if $\lambda^{2} \Delta \phi \gg 1$. Faraday thick sources are extended in $\phi$. They are substantially depolarized at $\lambda^{2}$. Remember that whether a source is Faraday thick or Faraday thin is wavelength dependent. See Fig. 1 and Appendix B for examples.

The rotation measure is commonly defined as the slope of a polarization angle $\chi$ versus $\lambda^{2}$ plot:

$\mathrm{RM}=\frac{\mathrm{d} \chi\left(\lambda^{2}\right)}{\mathrm{d} \lambda^{2}}$

where

$\chi=\frac{1}{2} \tan ^{-1} \frac{U}{Q}$.

Burn (1966) also introduces the complex Faraday dispersion function $F(\phi)$, which is defined through

$P\left(\lambda^{2}\right)=\int_{-\infty}^{+\infty} F(\phi) \mathrm{e}^{2 \mathrm{i} \phi \lambda^{2}} \mathrm{~d} \phi$.

$F(\phi)$ is the complex polarized surface brightness per unit Faraday depth, and $P\left(\lambda^{2}\right)=p\left(\lambda^{2}\right) I\left(\lambda^{2}\right)$ is the complex polarized surface brightness. Burn assumes that $F(\phi)$ is independent of frequency. In Sect. 3 we investigate to what extent this assumption can be relaxed.

$P$ can be written as

$P=\|p\| I \mathrm{e}^{2 \mathrm{i} \chi}$,

or equivalently,

$P=p I=Q+\mathrm{i} U$.

Equation (6) is very similar to a Fourier transform. A fundamental difference is that $P\left(\lambda^{2}\right)$ only has physical meaning for $\lambda^{2} \geq 0$. Because $P$ cannot be measured at $\lambda^{2}<0$, Eq. (6) is only invertible if one makes some assumptions on the value of $P$ at $\lambda^{2}<0$ based on its value at $\lambda^{2} \geq 0$ (Burn 1966). An example is that $P$ is Hermitian. This corresponds to assuming that $F(\phi)$ is strictly real. Burn showed that his approach worked by performing the inversion successfully on the Crab nebula. However, in his derivation of the inverse, he did not consider the effect of incomplete sampling of the domain $\lambda^{2}>0$. In Sect. 2 we treat a generalization of Eq. (6) that is both invertible and takes arbitrary sampling of $\lambda^{2}$ space into account. We refer to Appendix B for an example illustrating the effect of different sampling domains.

If there is only one source along the line of sight, which in addition has no internal Faraday rotation, and does not suffer from beam depolarization, then the Faraday depth of that source is equal to its rotation measure at all wavelengths:

$\chi\left(\lambda^{2}\right)=\chi_{0}+\phi \lambda^{2}$.

In general, however, this is not the case (e.g. Vallee 1980).

A simple example illustrates this. Imagine a classical double radio galaxy, of which the lobe closest to us is at a Faraday depth of $\phi_{1} \mathrm{rad} \mathrm{m}^{-2}$. The lobe itself is Faraday thin and has an intrinsic polarized flux density of $0.25 \mathrm{Jy}$ beam $^{-1}$ (positive Stokes $Q$ ). At low frequencies, there is usually some polarized Galactic foreground emission between us and the radio galaxy. The Galactic foreground is modelled as a uniform slab with a constant, uniform magnetic field. The total integrated polarized surface brightness of the Galactic foreground at $\lambda=0$ is $1 \mathrm{Jy} \mathrm{beam}^{-1}$ (positive Stokes $Q$ ). The Faraday dispersion function $F(\phi)$ is a top hat function:

$F(\phi)= \begin{cases}\left(2 \phi_{\mathrm{fg}}\right)^{-1} & -\phi_{\mathrm{fg}} \leq \phi \leq+\phi_{\mathrm{fg}} \\ 0 & \text { elsewhere. }\end{cases}$

In order to let the foreground emission start at $\phi \neq 0$ we assume a Faraday rotating, but non emitting medium between us and 
the Galactic foreground emission. For the sake of simplicity we assume that the total intensity spectra of both sources are flat. The complex polarized flux of this configuration is

$$
\begin{aligned}
P\left(\lambda^{2}\right) & =P_{\mathrm{fg}}\left(\lambda^{2}\right)+P_{1}\left(\lambda^{2}\right) \\
& =\left(2 \phi_{\mathrm{fg}}\right)^{-1} \int_{-\phi_{\mathrm{fg}}}^{+\phi_{\mathrm{fg}}} \mathrm{e}^{2 \mathrm{i} \phi \lambda^{2}} \mathrm{~d} \phi+\frac{1}{4} \mathrm{e}^{2 \mathrm{i} \phi_{1} \lambda^{2}} \\
& =\frac{\sin 2 \phi_{\mathrm{fg}} \lambda^{2}}{2 \phi_{\mathrm{fg}} \lambda^{2}}+\frac{1}{4} \cos \left(2 \phi_{1} \lambda^{2}\right)+\frac{1}{4} \mathrm{i} \sin \left(2 \phi_{1} \lambda^{2}\right),
\end{aligned}
$$

where the first term is the contribution of the Galactic foreground and the last two terms are due to the radio lobe. The first term in Eq. (13) is also called the Burn depolarization function. The result for the uniform slab, and results for several other simple models can be found in Gardner \& Whiteoak (1966) and Burn (1966). $P_{\mathrm{fg}}\left(\lambda^{2}\right)$ is real because the $F(\phi)$ of the Galactic foreground emission is symmetric around 0 .

Figure 1 plots $\chi,\|P\|$, and $Q_{\mathrm{fg}}$ for Eq. (13). $Q_{\mathrm{fg}}$ is the real part of $P_{\mathrm{fg}}$. We have taken $\phi_{1}=+10 \mathrm{rad} \mathrm{m}^{-2}$ and $\phi_{\mathrm{fg}}=$ $2 \mathrm{rad} \mathrm{m}^{-2}$. At low $\lambda^{2}$, the foreground dominates over the lobe, forcing Stokes $Q$ of the sum of the polarizations to be positive, while $U$ can be both positive and negative. In this regime, $\chi$ oscillates around zero. However, when the foreground is significantly depolarized, the lobe starts to dominate the total $(Q, U)$ vector. This point is reached somewhere near $\lambda^{2}=0.55 \mathrm{~m}^{2}$. From there on the total $(Q, U)$ vector runs through all four quadrants. As the polarized flux of the foreground vanishes, the total polarization angle approaches more and more a straight line corresponding to a $\mathrm{RM}$ of $+10 \mathrm{rad} \mathrm{m}^{-2}$.

Figure 2 shows an example of a fairly complex line of sight. There are three areas with polarized emission (A, B, and C), of which two (A and B) also have internal Faraday rotation. The middle panel shows the non-monotonic relation between Faraday depth and physical depth. Although area B is larger in physical depth, area A is larger in Faraday depth due to the high absolute value of $n_{\mathrm{e}} \boldsymbol{B}_{\|}$.

A physical interpretation of this example would be that region $\mathrm{A}$ and its adjacent rotation-only areas reside in our Galaxy, area $\mathrm{B}$ and its neighboring rotation-only areas are a galaxy cluster, and area $\mathrm{C}$ represents a collection of distant polarized sources without any internal Faraday rotation of their own. Line of sight 1 goes through the cluster, while line of sight 2 just misses it. This causes $\mathrm{C}$ to be at different Faraday depth in the two lines of sight.

Because of the Fourier nature of both Eq. (6) and radio synthesis imaging, there exist many analogies between the two. Examples are $u v$ plane sampling versus $\lambda^{2}$ sampling and synthesized beam versus RMTF. Therefore we prefer to call the process of inversion "Rotation Measure synthesis" ("RM-synthesis" for short).

Similar methods have recently been applied to pulsar observations (Mitra et al. 2003; Weisberg et al. 2004). de Bruyn (1996) applied the method for the first time to an entire field of view. He also introduced the concept of a Rotation Measure Transfer Function (RMTF, see also Sect. 2 of this work). When applied to a complete field of view instead of just one line of sight, the output of a RM-synthesis is a so-called "RM-cube". The RM-cube has axes $\alpha, \delta$, and $\phi$. It is the Faraday rotation
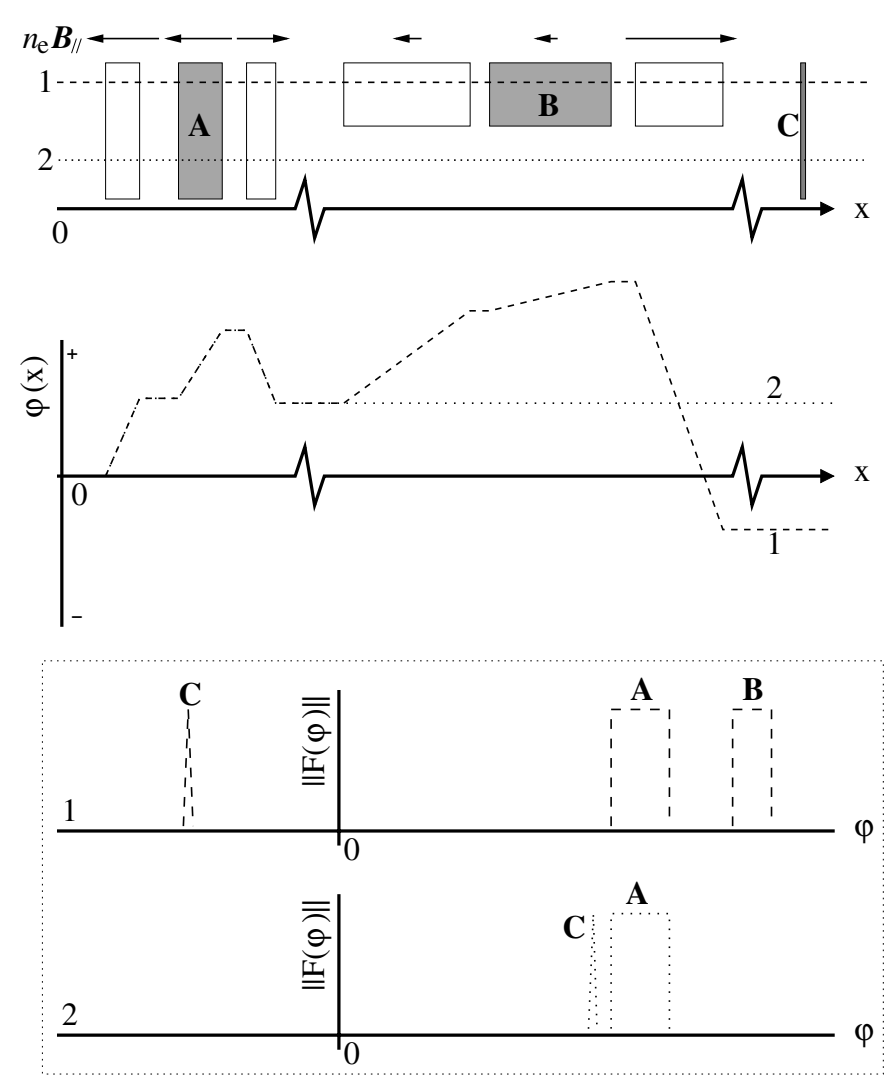

Fig. 2. Cartoon sketching the relation between emission, $n_{\mathrm{e}} \boldsymbol{B}_{\|}$, Faraday depth $\phi$, location $x$, and observed Faraday spectrum. The top panel depicts the physical situation. The arrows represent $n_{\mathrm{e}} \boldsymbol{B}_{\|}$. Longer arrows mean larger $\left\|n_{\mathrm{e}} \boldsymbol{B}_{\|}\right\|$. The direction of the arrow indicates the direction of the parallel component of the magnetic field. The $x$ coordinate represents physical distance from the observer. The observer is located at the far left of the plots. The $x$ axis is severely compressed in two places. Empty areas have neither emission nor rotation. White blocks represent areas with only Faraday rotation. Grey areas with an arrow have both emission and rotation (area A and B) and grey areas without an arrow have only emission (area C). There are two lines of sight, labelled 1 and 2. Line of sight 1 goes through areas A, B, and C. Line of sight 2 misses area B as well as the adjacent non emitting Faraday rotating white boxes. The middle panel plots Faraday depth $\phi$ as a function of physical distance $x$ for both lines of sight. The bottom panel shows $\|F(\phi)\|$, the observed polarized surface brightness $\left(\mathrm{rad} \mathrm{m}^{-2}\right)^{-1}$, for both lines of sight. The peaks in the spectra are labelled with the associated areas.

equivalent of a $21 \mathrm{~cm}$ line cube. The application to wide fields allowed the discovery of widespread, very faint polarized emission associated with the Perseus cluster (de Bruyn \& Brentjens 2005).

Modern correlator backends, like the ones installed at the WSRT, the GMRT, and the ATCA and the one to be installed at the EVLA deliver the visibilities in many (32 to 1024) narrow channels across a wide band (typically 16 to $160 \mathrm{MHz}$ ). The narrow channels move the bandwidth depolarization limit to much higher rotation measures. The wider bands yield very high sensitivities if the full bandwidth can be used. Thanks to these backends RM-synthesis has finally become a practical, even necessary observing method. 
Section 2 discusses the generally incomplete sampling of $\lambda^{2}>0$. We formally derive the RMTF. Section 3 treats modifications to the assumption that $F(\phi)$ is frequency independent. In Sect. 4 we treat the relation between the RMTF and $n \pi$ ambiguities in traditional RM fitting. Section 5 describes RM-synthesis with Stokes $Q$ or $U$ only. Section 6 gives advice on designing Faraday rotation experiments, taking the findings of this work into account. Section 7 concludes this work. Appendix A expands on error estimation in RM work and Appendix B treats an example simulation illustrating a few important concepts presented in this work.

\section{Derivation}

The goal of this section is to approximate $F(\phi)$ by Fourier inverting a generalized version of Eq. (6). Table 1 summarizes the symbols that are used throughout this paper. We generalize Eq. (6) by introducing the weight function $W\left(\lambda^{2}\right) . W\left(\lambda^{2}\right)$ is also called the sampling function. It is nonzero at all $\lambda^{2}$ points where measurements are taken. It is zero elsewhere. Obviously, $W\left(\lambda^{2}\right)=0$ for $\lambda^{2}<0$ because of the lack of measurements there. The observed polarized flux density, or surface brightness in the case of spatially extended sources, is

$\tilde{P}\left(\lambda^{2}\right)=W\left(\lambda^{2}\right) P\left(\lambda^{2}\right)$.

The tilde is used to indicate observed or reconstructed quantities. Substituting Eq. (6) gives

$\tilde{P}\left(\lambda^{2}\right)=W\left(\lambda^{2}\right) \int_{-\infty}^{+\infty} F(\phi) \mathrm{e}^{2 \mathrm{i} \phi \lambda^{2}} \mathrm{~d} \phi$.

Equation (6) is very similar to the Fourier transform pair

$$
\begin{aligned}
& f(x)=\int_{-\infty}^{+\infty} F(t) \mathrm{e}^{-2 \pi \mathrm{i} x t} \mathrm{~d} t \\
& F(t)=\int_{-\infty}^{+\infty} f(x) \mathrm{e}^{2 \pi \mathrm{i} x t} \mathrm{~d} x .
\end{aligned}
$$

Substituting $\lambda^{2}=\pi u$ in Eq. (15) gives

$\tilde{P}(\pi u)=W(\pi u) \int_{-\infty}^{+\infty} F(\phi) \mathrm{e}^{2 \pi \mathrm{i} \phi u} \mathrm{~d} \phi$.

We define the function

$R(\phi)=\frac{\int_{-\infty}^{+\infty} W(\pi u) \mathrm{e}^{-2 \pi \mathrm{i} \phi u} \mathrm{~d} u}{\int_{-\infty}^{+\infty} W(\pi u) \mathrm{d} u}$,

which is normalized to unity at $\phi=0$. The inverse is

$W(\pi u)=\left(\int_{-\infty}^{+\infty} W(\pi u) \mathrm{d} u\right) \int_{-\infty}^{+\infty} R(\phi) \mathrm{e}^{2 \pi \mathrm{i} \phi u} \mathrm{~d} \phi$.

Equations (18) and (20) are now combined. Application of the convolution theorem to the result gives

$F(\phi) * R(\phi)=\frac{\int_{-\infty}^{+\infty} \tilde{P}(\pi u) \mathrm{e}^{-2 \pi \mathrm{i} \phi u} \mathrm{~d} u}{\int_{-\infty}^{+\infty} W(\pi u) \mathrm{d} u}$

\begin{tabular}{|c|c|}
\hline Symbol & Description \\
\hline$\chi$ & Polarization angle ( $\mathrm{N}$ through $\mathrm{E})$ \\
\hline$\chi_{0}$ & Polarization angle at $\lambda=0$ \\
\hline$v$ & Frequency \\
\hline$\delta v$ & Channel width in frequency \\
\hline$v_{\mathrm{c}}$ & Central frequency of a channel \\
\hline$\lambda$ & Wavelength \\
\hline$\lambda_{0}$ & $\begin{array}{l}\text { Wavelength to which all polarization vectors are } \\
\text { derotated }\end{array}$ \\
\hline$\lambda_{\mathrm{c}}^{2}$ & Central wavelength squared of a channel \\
\hline$\delta \lambda^{2}$ & Channel width in wavelength squared \\
\hline$\Delta \lambda^{2}$ & $\begin{array}{l}\text { Total bandwidth in wavelength squared. } \Delta \lambda^{2}= \\
\lambda_{\max }^{2}-\lambda_{\min }^{2}\end{array}$ \\
\hline$\phi$ & Faraday depth \\
\hline$\delta \phi$ & FWHM of the main peak of the RMTF \\
\hline $\mathrm{RM}$ & Rotation measure \\
\hline$W\left(\lambda^{2}\right)$ & Weight function \\
\hline$w_{i}$ & Weight of the $i$ th data point \\
\hline$K$ & $\begin{array}{l}\text { One over the integral of } W \text { or one over the sum of } \\
\text { weights }\end{array}$ \\
\hline$F(\phi)$ & $\begin{array}{l}\text { Faraday dispersion function without spectral depen- } \\
\text { dence }\end{array}$ \\
\hline$\tilde{F}(\phi)$ & Reconstructed approximation to $F(\phi)$ \\
\hline$F\left(\phi, \lambda^{2}\right)$ & General form of the Faraday dispersion function \\
\hline$f(\phi)$ & $F\left(\phi, \lambda^{2}\right) / s\left(\lambda^{2}\right)$ \\
\hline$s\left(\lambda^{2}\right)$ & $\begin{array}{l}\text { Spectral dependence in } I, \text { normalized to unity at } \lambda^{2}= \\
\lambda_{0}^{2}\end{array}$ \\
\hline$\alpha$ & Frequency spectral index \\
\hline$P\left(\lambda^{2}\right)$ & Complex polarized surface brightness \\
\hline$\tilde{P}\left(\lambda^{2}\right)$ & Observed $P: W\left(\lambda^{2}\right) P\left(\lambda^{2}\right)$ \\
\hline$p\left(\lambda^{2}\right)$ & Complex polarization fraction $P\left(\lambda^{2}\right) / I\left(\lambda^{2}\right)$ \\
\hline$R(\phi)$ & Rotation Measure Transfer Function (RMTF) \\
\hline $\boldsymbol{B}$ & Magnetic induction \\
\hline$r$ & Position vector \\
\hline$n_{\mathrm{e}}$ & Thermal electron density \\
\hline$\gamma$ & $\begin{array}{l}\text { Spectral index of the relativistic electron energy dis- } \\
\text { tribution }\end{array}$ \\
\hline $\mathfrak{R} z$ & Real part of $z$ \\
\hline $\mathfrak{J} z$ & Imaginary part of $z$ \\
\hline$\rho$ & $\begin{array}{l}\text { Merit function for traditional linear least squares fit- } \\
\text { ting of rotation measures. Defined in Eq. (49) }\end{array}$ \\
\hline$\sigma$ & rms noise in a single channel map \\
\hline$\sigma_{\mathrm{Q}}, \sigma_{\mathrm{U}}$ & rms noise in single $Q$ or $U$ channel maps \\
\hline$\sigma_{\mathrm{P}}, \sigma_{\chi}$ & $\begin{array}{l}\text { Standard error of }\|P\| \text { and } \chi \text { in individual channel } \\
\text { maps }\end{array}$ \\
\hline$\sigma_{\phi}, \sigma_{\chi 0}$ & $\begin{array}{l}\text { Standard error in Faraday depth and position angle } \\
\text { at } \lambda=0\end{array}$ \\
\hline$\sigma_{\lambda^{2}}$ & $\begin{array}{l}\text { Standard deviation of the distribution of } \lambda^{2} \text { values } \\
\text { that are sampled. This is a measure of the effective } \\
\text { width of the } \lambda^{2} \text { sampling }\end{array}$ \\
\hline$\delta(x)$ & Dirac delta function \\
\hline
\end{tabular}

Table 1. List of symbols. 


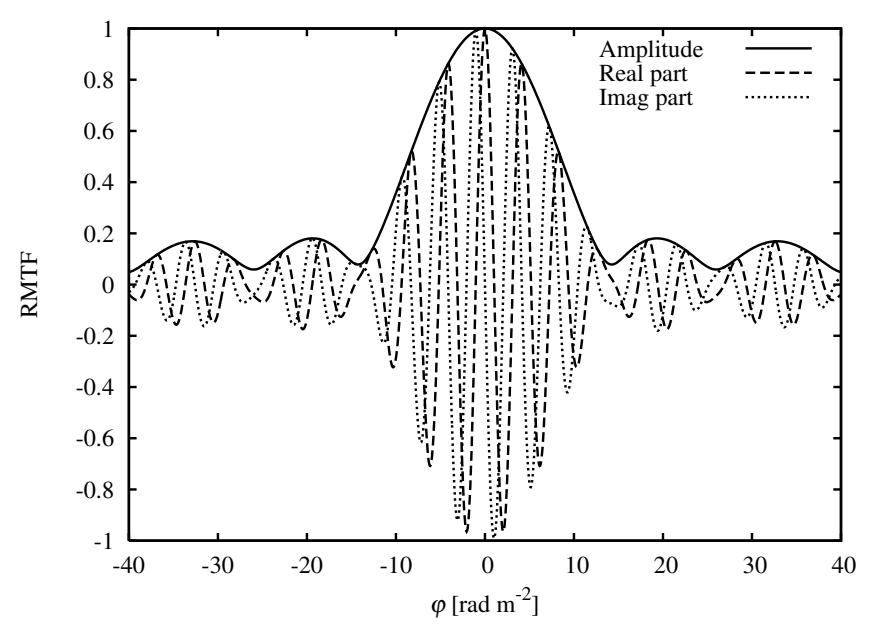

Fig. 3. RMTF of a $92 \mathrm{~cm}$ dataset taken with the Westerbork Synthesis Radio Telescope. There are 126 usable channels in the dataset. All $(Q, U)$ vectors have been derotated to $\lambda_{0}^{2}=0$. Note the rapid rotation of the RMTF, making it difficult to measure accurate polarization angles in a sampled RM cube.

where $*$ denotes convolution. After back substituting $u=\lambda^{2} / \pi$, we obtain

$$
\begin{aligned}
\tilde{F}(\phi)=F(\phi) * R(\phi) & =K \int_{-\infty}^{+\infty} \tilde{P}\left(\lambda^{2}\right) \mathrm{e}^{-2 \mathrm{i} \phi \lambda^{2}} \mathrm{~d} \lambda^{2} \\
R(\phi) & =K \int_{-\infty}^{+\infty} W\left(\lambda^{2}\right) \mathrm{e}^{-2 \mathrm{i} \phi \lambda^{2}} \mathrm{~d} \lambda^{2} \\
K & =\left(\int_{-\infty}^{+\infty} W\left(\lambda^{2}\right) \mathrm{d} \lambda^{2}\right)^{-1} .
\end{aligned}
$$

$\tilde{F}(\phi)$ is an approximate reconstruction of $F(\phi)$. More precisely, it is $F(\phi)$ convolved with $R(\phi)$ after Fourier filtering by the weight function $W\left(\lambda^{2}\right)$. The quality of the reconstruction depends mainly on the weight function $W\left(\lambda^{2}\right)$. A more complete coverage of $\lambda^{2}$ space improves the reconstruction. Fewer holes in the $\lambda^{2}$ sampling reduce the side lobes of $R(\phi)$, while covering a larger range of $\lambda^{2}$ increases the resolution in $\phi$ space. We return to these statements in the following sections. We call $R(\phi)$ the rotation measure transfer function (RMTF).

The above set of equations is not yet our final result. Figure 3 displays the rotation measure transfer function corresponding to the $\lambda^{2}$ sampling of our Perseus data set (de Bruyn $\&$ Brentjens 2005). It only shows a small part of the RMTF close to the peak. The response function displays a rapid rotation of the (real, imaginary) vector. Because one usually samples $\phi$ space at finite intervals, this rotation makes it very difficult to correctly estimate the polarization angle at or near the maximum of $\|F(\phi)\|$. If the Faraday depth of a frame is only a tenth of the width of the RMTF away from the actual Faraday depth of the source, the (real, imaginary) vector may already be rotated by several tens of degrees.

Equations (22) and (23) correspond to derotating all polarization vectors back to their position at $\lambda=0$. At first this appears sensible, because the polarization vector at $\lambda=0$ is directly related to the electric field vector in the plane of the sky without any Faraday rotation. Nevertheless no information is lost by derotating to some other common $\lambda_{0}^{2} \neq 0$.
The more general versions of Eqs. (22) and (23) are

$$
\begin{aligned}
& \tilde{F}(\phi)=K \int_{-\infty}^{+\infty} \tilde{P}\left(\lambda^{2}\right) \mathrm{e}^{-2 \mathrm{i} \phi\left(\lambda^{2}-\lambda_{0}^{2}\right)} \mathrm{d} \lambda^{2} \\
& R(\phi)=K \int_{-\infty}^{+\infty} W\left(\lambda^{2}\right) \mathrm{e}^{-2 \mathrm{i} \phi\left(\lambda^{2}-\lambda_{0}^{2}\right)} \mathrm{d} \lambda^{2} .
\end{aligned}
$$

This is effectively an application of the shift theorem of Fourier theory. Because the shift theorem only affects the argument, and not the absolute value of the resulting complex function, nothing changes in the amplitude of the RMTF. Equations (25) and (15) form a Fourier pair that enables us to transform polarization information from $\lambda^{2}$ space to $\phi$ space and back. The function $R(\phi)$ is our final form of the rotation measure transfer function (RMTF). It is a complex valued function. The real part corresponds to the response of the transform parallel to the $(Q, U)$ vector at $\lambda=\lambda_{0}$ and the imaginary part corresponds to the response orthogonal to it. Assume that one has a Faraday thin source at Faraday depth $\phi_{0}$, of which the polarization angle is $45^{\circ}$ (all polarized emission is in positive $U$ ) at $\lambda^{2}=\lambda_{0}^{2}$. Whenever $R\left(\phi-\phi_{0}\right)$ is real and positive, $\tilde{F}(\phi)$ would be imaginary (all polarized flux in positive Stokes $U$ ). If, however $R(\phi)$ is positive imaginary, $\tilde{F}(\phi)$ would be real and negative (all polarized flux in negative Stokes $Q$. This is important if $\phi \neq \phi_{0}$, that is, when the Faraday depth of the source does not match the Faraday depth that was chosen for evaluation of $\tilde{F}(\phi)$.

The simplest way to see this is to consider the case when $F(\phi)=P\left(\lambda_{0}^{2}\right) \delta\left(\phi-\phi_{\text {source }}\right)$. This changes the convolution in Eq. (25) into a multiplication. Hence the result of the righthand side of Eq. (25) can be written as

$$
\tilde{F}(\phi)=P\left(\lambda_{0}^{2}\right) R\left(\phi-\phi_{\text {source }}\right) \text {. }
$$

If $R$ is imaginary, it can be written as $\|R\| \mathrm{e}^{ \pm \mathrm{i} \pi / 2}$. Multiplication of a complex number with $\mathrm{e}^{ \pm \mathrm{i} \pi / 2}$ corresponds to a rotation in the complex plane of $\pm \pi / 2$, hence the apparent polarization angle would have rotated by $45^{\circ}$ relative to the actual polarization angle at $\lambda^{2}=\lambda_{0}^{2}$.

Ideally, the response in the entire main peak of the RMTF should be parallel to the actual polarization vector at $\lambda_{0}$. The best way of achieving that is keeping the orthogonal response as close to zero as possible. We set the derivative of the imaginary part at $\phi=0$ to zero:

$$
\begin{aligned}
& 0=\left.\frac{\partial \mathfrak{I R}(\phi)}{\partial \phi}\right|_{\phi=0} \\
& 0=-\left.K \frac{\partial}{\partial \phi} \int_{-\infty}^{+\infty} W\left(\lambda^{2}\right) \sin 2 \phi\left(\lambda^{2}-\lambda_{0}^{2}\right) \mathrm{d} \lambda^{2}\right|_{\phi=0} \\
& 0=-\left.K \int_{-\infty}^{+\infty} W\left(\lambda^{2}\right) 2\left(\lambda^{2}-\lambda_{0}^{2}\right) \cos 2 \phi\left(\lambda-\lambda_{0}^{2}\right) \mathrm{d} \lambda^{2}\right|_{\phi=0} \\
& 0=\int_{-\infty}^{+\infty} W\left(\lambda^{2}\right)\left(\lambda^{2}-\lambda_{0}^{2}\right) \mathrm{d} \lambda^{2} \\
& \lambda_{0}^{2}=\frac{\int_{-\infty}^{+\infty} W\left(\lambda^{2}\right) \lambda^{2} \mathrm{~d} \lambda^{2}}{\int_{-\infty}^{\infty} W\left(\lambda^{2}\right) \mathrm{d} \lambda^{2}}
\end{aligned}
$$

hence $\lambda_{0}^{2}$ should be made equal to the weighted average of the observed $\lambda^{2}$. 


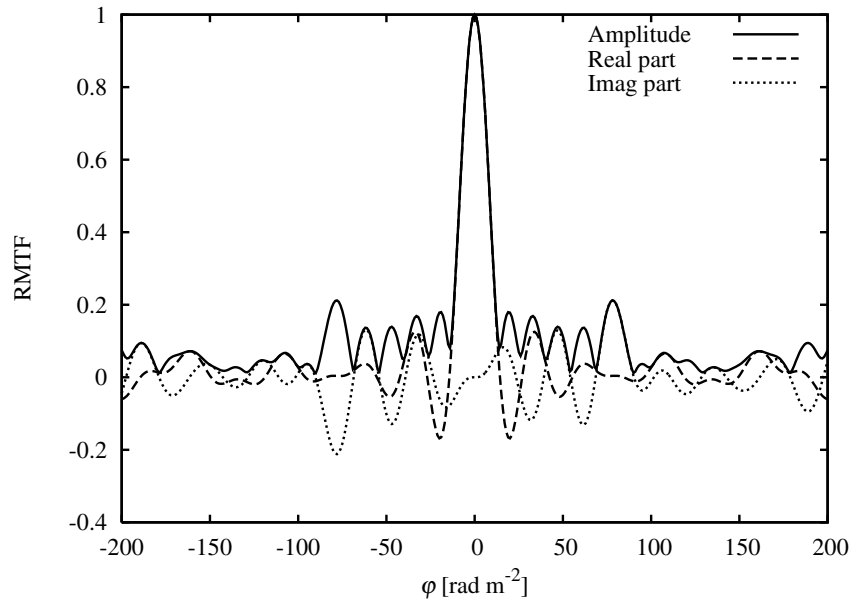

Fig. 4. RMTF of the same dataset as described in Fig. 3. This time, however, all $\boldsymbol{P}$ vectors have been derotated to the average $\lambda^{2}$. It is seen that the imaginary part remains almost constant within the central peak of the RMTF.

A drawback of having $\lambda_{0}^{2} \neq 0$ is that the polarization angle that one derives still needs to be transformed to a polarization angle at $\lambda^{2}=0$, if one wants information on the orientation of the electric field direction in the source. In case of a high $S / N$ ratio, this is very easy:

$\chi_{0}=\chi\left(\lambda_{0}^{2}\right)-\phi \lambda_{0}^{2}$

However, if the signal to noise ratio is low, the uncertainty in $\phi$ usually prevents accurate derotation to $\lambda^{2}=0$. The advantage of derotating to the weighted average $\lambda^{2}$ is that one can still properly analyze spatial coherence of polarization angles in a spatially extended source at a certain Faraday depth.

Figure 4 shows the same RMTF as Fig. 3, except that $\lambda_{0}^{2}$ is set to the weighted average $\lambda^{2}$. The improvement with respect to the orthogonal response is evident. The response function is almost completely real between the first minima. The only drawback is that one cannot convert the observed polarization angle at $\lambda_{0}$ to a $\boldsymbol{E}$ vector in a straightforward way. In order to accomplish reliable derotation to $\lambda=0$, one needs a sufficiently high $S / N$ ratio to determine the Faraday depth with an accuracy well within the full width at half maximum (FWHM) of the RMTF. This is not a problem for bright sources that are already detected in individual channels, but for faint emission that is only detectable after RM-synthesis, one cannot usually do this. These signal to noise statements are quantified in Sect. 4 and Appendix A.

In most correlators, all channels have equal bandwidth $\delta v$, centred around $v_{\mathrm{c}}$, the central frequency of the channel. Our prime coordinate is $\lambda^{2}$, not $v$. If we assume a top hat channel bandpass, we have for every channel:

$\lambda_{\mathrm{c}}^{2} \approx \frac{c^{2}}{v_{\mathrm{c}}^{2}}\left(1+\frac{3}{4}\left(\frac{\delta v}{v_{\mathrm{c}}}\right)^{2}\right)$

and

$\delta \lambda^{2} \approx \frac{2 c^{2} \delta v}{v_{\mathrm{c}}^{3}}\left(1+\frac{1}{2}\left(\frac{\delta v}{v_{\mathrm{c}}}\right)^{2}\right)$.
Of course the channel bandpass is usually not a top hat function, but rather a sinc if no taper was applied before the timeto-frequency transform, or something in between if other tapers like Hanning or Kaiser-Bessel are used. These differences are hardly important as long as $\delta v / v_{\mathrm{c}} \ll 1$, which is usually the case.

If $\phi \delta \lambda^{2} \ll 1$ for all channels, we may approximate the integrals in Eqs. (25) and (26) by sums:

$$
\begin{aligned}
& \tilde{F}(\phi) \approx K \sum_{i=1}^{N} \tilde{P}_{i} \mathrm{e}^{-2 \mathrm{i} \phi\left(\lambda_{i}^{2}-\lambda_{0}^{2}\right)} \\
& R(\phi) \approx K \sum_{i=1}^{N} w_{i} \mathrm{e}^{-2 \mathrm{i} \phi\left(\lambda_{i}^{2}-\lambda_{0}^{2}\right)} \\
& K=\left(\sum_{i=1}^{N} w_{i}\right)^{-1} .
\end{aligned}
$$

In these equations, $\lambda_{i}^{2}$ is $\lambda_{\mathrm{c}}^{2}$ of channel $i, \tilde{P}_{i}=\tilde{P}\left(\lambda_{i}^{2}\right)=w_{i} P\left(\lambda_{i}^{2}\right)$, $w_{i}=W\left(\lambda_{i}^{2}\right)$, and $K$ has become the sum of all weights. We have implemented Eqs. (36), (37), and (38) in our RM-synthesis software.

\section{Spectral dependence}

In this section we investigate the effect of the emission spectrum of a source on the method. We start with the most general case of an arbitrary spectrum at each Faraday depth. We substitute

$F(\phi)=F\left(\phi, \lambda^{2}\right)$

in Eq. (15)

$\tilde{P}\left(\lambda^{2}\right)=W\left(\lambda^{2}\right) \int_{-\infty}^{+\infty} F\left(\phi, \lambda^{2}\right) \mathrm{e}^{2 \mathrm{i} \phi \lambda^{2}} \mathrm{~d} \phi$.

In general, this equation is not invertible, except in cases such as:

- $F\left(\phi, \lambda^{2}\right)$ can be written as a product of independent functions, i.e. $F\left(\phi, \lambda^{2}\right)=f(\phi) s\left(\lambda^{2}\right)$;

- $F\left(\phi, \lambda^{2}\right)=f(\phi) \delta\left(\phi-\phi_{0}\right) s\left(\lambda^{2}\right)$;

- the spectrum is a power law with $\alpha \propto \phi$.

$s\left(\lambda^{2}\right)$ is the spectral dependence

$s\left(\lambda^{2}\right)=\frac{I\left(\lambda^{2}\right)}{I\left(\lambda_{0}^{2}\right)}$.

The third case, although invertible, is highly non-physical. The inversion of the first case is trivial:

$F\left(\phi, \lambda^{2}\right)=f(\phi) s\left(\lambda^{2}\right)$

$\tilde{P}\left(\lambda^{2}\right)=W\left(\lambda^{2}\right) \int_{-\infty}^{+\infty} f(\phi) s\left(\lambda^{2}\right) \mathrm{e}^{2 \mathrm{i} \phi \lambda^{2}} \mathrm{~d} \phi$

$\frac{\tilde{P}\left(\lambda^{2}\right)}{s\left(\lambda^{2}\right)}=\tilde{p}\left(\lambda^{2}\right) I\left(\lambda_{0}^{2}\right)=W\left(\lambda^{2}\right) \int_{-\infty}^{+\infty} f(\phi) \mathrm{e}^{2 \mathrm{i} \phi \lambda^{2}} \mathrm{~d} \phi$.

This is equivalent to Eq. (15) divided by $s\left(\lambda^{2}\right)$, and we have already shown that Eq. (15) is invertible. 
The second case is a specialization of the first case. Equation (44) reduces to Eq. (15) in case of a flat spectrum. The approximate Faraday dispersion function compensated for a non-flat spectrum is given by:

$\tilde{f}(\phi)=K \int_{-\infty}^{+\infty} W\left(\lambda^{2}\right) \frac{P\left(\lambda^{2}\right)}{s\left(\lambda^{2}\right)} \mathrm{e}^{-2 \mathrm{i} \phi\left(\lambda^{2}-\lambda_{0}^{2}\right)} \mathrm{d} \lambda$,

or, if $W\left(\lambda^{2}\right)$ can be approximated by a sum of Dirac $\delta$ functions:

$\tilde{f}(\phi) \approx K \sum_{i=1}^{N} \frac{\tilde{P}_{i}}{s_{i}} \mathrm{e}^{-2 \mathrm{i} \phi\left(\lambda_{i}^{2}-\lambda_{0}^{2}\right)}$,

where $s_{i}=s\left(\lambda_{i}^{2}\right)$ and $\tilde{f}(\phi)=f(\phi) * R(\phi)$.

Equation (42) applies only in some very specific scenarios. It holds for example in optically thin synchrotron-emitting and Faraday-rotating clouds that have the same relativistic electron energy distribution throughout the cloud. It also holds if multiple optically thin clouds along the line of sight happen to have the same spectral dependence. Optically thin synchrotron radiation has a spectrum that is proportional to $v^{\alpha}$ over a large range of frequencies (Conway et al. 1963). For most sources, $\alpha$ is in the range $\langle-1.5,-0.5\rangle$. In extreme cases the spectral index of optically thin emission can approach 0 (e.g. the Crab nebula) or -3 (for halo or relic sources in galaxy clusters).

In general, spectral indices vary across a map. One can of course easily correct for the spectra of sources that are reliably detected in individual channel maps. This is impossible for sources that are much fainter and only show up after averaging the full band. For those objects it makes sense to estimate some "average" spectral index and apply that to the entire map.

What is the effect of using the wrong spectral index in correcting for the spectrum of a single source along the line of sight? The contributions of multiple sources along the line of sight is simply the sum of their individual responses. Because the spectrum is an amplitude only effect, it has no influence on the location of the maximum of the Faraday dispersion function of the source. Therefore its derived Faraday depth is unaffected. It does distort the RMTF associated with the source at points away from the main peak. This complicates deconvolution algorithms slightly.

Figure 5 gives the Faraday dispersion functions of Faraday thin model sources with spectral indices -3 to 0 . It is seen that the largest effect occurs close to the nulls of the RMTF. The difference between $\alpha=-3$ and $\alpha=0$ is small over the $17 \%$ total frequency bandwidth in the simulation. It will not be noticeable if the emission has such low $S / N$ that it is invisible in individual channels. For comparison, the normalized $\tilde{F}(\phi)$ of a Faraday thick uniform slab model is included. The slab emits at $-1 \leq \phi \leq+1 \mathrm{rad} \mathrm{m}^{-2}$. It is seen that the effect of even a tiny amount of $\phi$ structure in the source is much larger than the effect of changing the spectral index by \pm 1 .

The general case of an arbitrary spectral dependence at multiple Faraday depths is not invertible. One can only recover the Faraday dispersion function if the spectral dependence is the same at all Faraday depths along the line of sight. One should then divide the observed polarization by the spectral dependence in $I$. Figure 5 shows that if the spectral index is estimated with an absolute uncertainty less than 1, the maximum

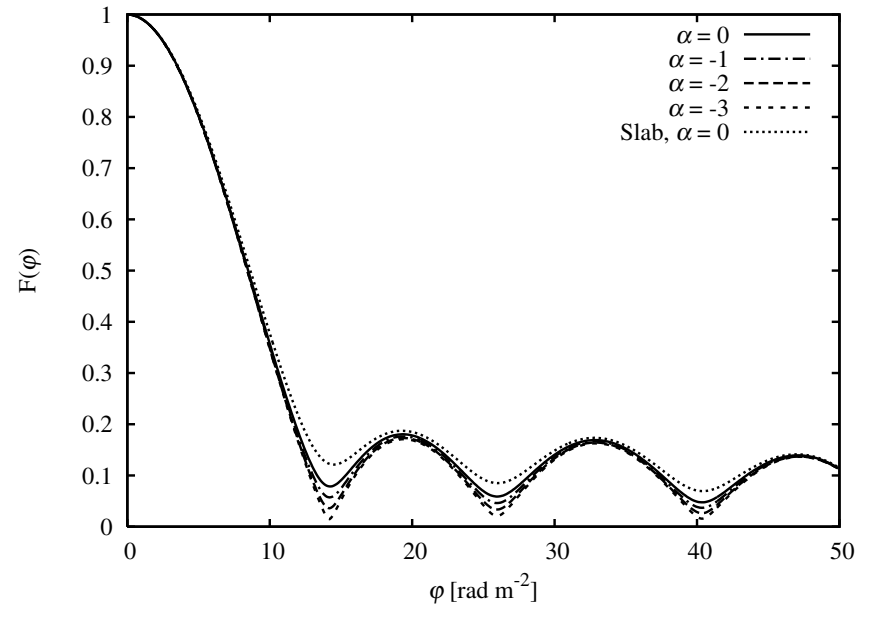

Fig. 5. Absolute value of the approximated Faraday dispersion function of several Faraday thin sources with different spectral indices. The $\lambda^{2}$ grid is the same previously mentioned 126 channel dataset. The overall bandwidth $\Delta v / v=17 \%$. Steeper spectra give deeper nulls. For comparison the normalized approximated Faraday dispersion function of a Faraday thick slab was included.

absolute error of the estimated flux density at a certain Faraday depth is less than $2-5 \%$ of the brightest emission along the line of sight. This accuracy is easily exceeded for sources that are visible in total intensity. Sources that have not been detected in total intensity should generally be assigned a spectral index of -1 . This worked very well in our observations of the Perseus cluster, where we see large, faint polarized features that have no detectable counterpart in total intensity (de Bruyn \& Brentjens 2005).

\section{4. $n \pi$ ambiguities and the RMTF}

The traditional way to compute the rotation measure of a source is to measure its polarization angle at several wavelengths and determine the slope of a straight line through the polarization angle as a function of $\lambda^{2}$. This method suffers from so-called $n \pi$ ambiguity problems. If only a few data points are available, there may exist multiple RM solutions that are equally good, but have the polarization angle of the data points wrapped around one or more turns. Complicated methods have been devised to attempt to circumvent these problems, some of which are quite successful. An example is the "Pacerman" method (Dolag et al. 2004; Vogt et al. 2004), which operates on images, and does a good job in finding and correcting $n \pi$ ambiguities using spatial continuity arguments.

In this section we show that the RMTF is an excellent indicator of possible $n \pi$ ambiguity problems. By analyzing the RMTF, one can take measures to minimize or even eradicate any potential $n \pi$ problems in the experiment in advance. We also show that using only RM-synthesis to determine Faraday depths is as accurate as traditional $\lambda^{2}$ fitting, but has the added value of straightforward $n \pi$ ambiguity problem detection.

We first consider traditional $\lambda^{2}$ fitting. This is done by linear least squares minimization of a merit function $\rho$. If the estimated errors of all points are equal, then the merit function 


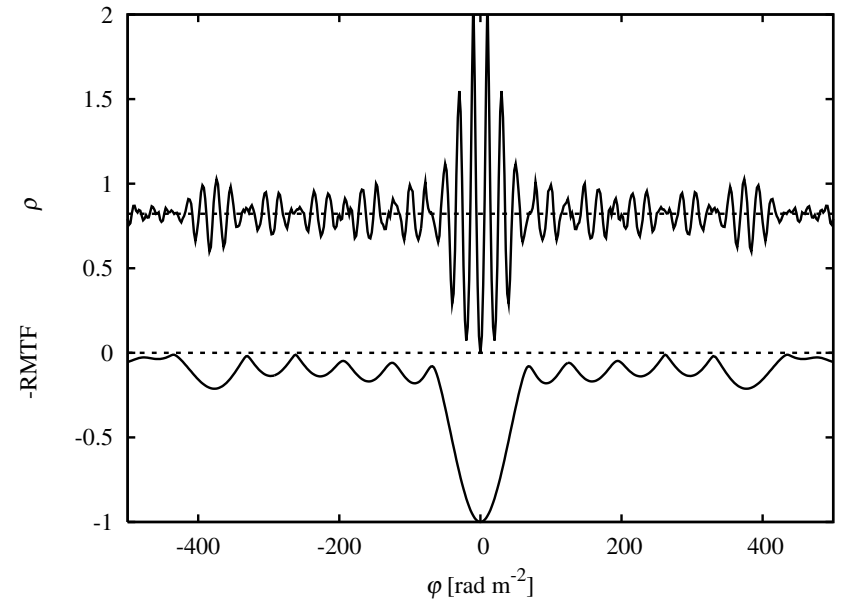

Fig. 6. Comparison of merit function $\rho$ (top) and $-\|R\|$ (bottom). The dotted line through $\rho$ is at $y=\pi^{2} / 12$.

looks like Eq. (49)

$z_{i}=\left\|\chi_{\mathrm{M}}\left(\mathrm{RM}, \chi_{0}, \lambda_{i}^{2}\right)-\chi_{i}\right\|$

$l_{i}= \begin{cases}z_{i} & z_{i}<\frac{1}{2} \pi \\ z_{i}-\pi & z_{i} \geq \frac{1}{2} \pi\end{cases}$

$\rho=N^{-1} \sum_{i=1}^{N} l_{i}^{2}$

$\chi_{\mathrm{M}}$ is a polarization angle computed by a model (for example Eq. (9)). $\chi_{i}$ is the observed polarization angle of the $i$ th data point. Both $\chi_{\mathrm{M}}$ and $\chi_{i}$ are modulo $\pi$. Equation (48) ensures that $l_{i} \in\left[-\frac{1}{2} \pi, \frac{1}{2} \pi\right\rangle$. For the sake of simplicity, we assume that we want to fit a source with a constant polarization angle of $0^{\circ}$. In that case Eq. (49) reduces to

$\rho=N^{-1} \sum_{i=1}^{N}\left(\left[\operatorname{RM} \lambda_{i}^{2}\right] \bmod \frac{\pi}{2}\right)^{2}$.

If the fit to the (noiseless) simulated data is perfect, $\rho=0$. When comparing Eq. (50) and Eq. (37), it is seen that $\rho=0$ if and only if $\left\|R\left(\phi-\phi_{0}\right)\right\|=1 . \phi_{0}$ denotes the Faraday depth of the source. In this simple case $\phi=\mathrm{RM}$. If $\left\|R\left(\phi-\phi_{0}\right)\right\|<1$, then $\rho>0$.

If the model RM is sufficiently different from the actual $\mathrm{RM}$, one expects the errors $l_{i}$ to be approximately uniformly distributed in the range $\left[-\frac{1}{2} \pi, \frac{1}{2} \pi\right\rangle$. Because the square is taken, this is equivalent to a uniform distribution in the range $\left[0, \frac{1}{2} \pi\right]$. The average value of $l_{i}^{2}$ is then given by

$\left\langle l_{i}^{2}\right\rangle=\frac{2}{\pi} \int_{0}^{\frac{1}{2} \pi} x^{2} \mathrm{~d} x=\frac{\pi^{2}}{12}$

The top graph of Fig. 6 plots $\rho$. The pattern of sample points is that of the 126 points used in Fig. 4. In this case, the width of the pattern is scaled to $\Delta \lambda^{2}=0.0459 \mathrm{rad} \mathrm{m}^{-2}$. The bottom graph is $-\left\|R\left(\phi-\phi_{0}\right)\right\|$. The dashed lines are at $y=0$ and $y=$ $\pi^{2} / 12$. It is seen that $\rho$ is indeed zero when $\|R\|=1$. Also, the average value at high RM is indeed equal to $\pi^{2} / 12$, especially when $\|R\|$ is close to zero. This implies that when $\|R\|$ is close to zero, $\phi \lambda^{2}$ is distributed uniformly between $-\frac{1}{2} \pi$ and $+\frac{1}{2} \pi$. Of course $\|R\|$ can be zero for other reasons, but due to the large number of points and the dense filling of $\Delta \lambda^{2}$, that is rather unlikely.

An interesting aspect seen in Fig. 6 is that the envelope of $\rho-\pi^{2} / 12$ looks like $-\|R\|$ when $\rho<\pi^{2} / 12$. Deep minima of $\rho$ are associated with high peaks in the RMTF. In fact, they appear to be approximately proportional to $\|R\|$. These deep minima are closely related to so-called $n \pi$ ambiguities in traditional RM measurements. They are points that fit the data (almost) equally well as the "true" solution.

The similarity between the envelope of $\rho$ and $-\|R\|$ is better demonstrated in Fig. 7. It shows both $\rho$ and $-\|R\|$ over a large range in $\phi$. The lefthand panel displays $\rho$ and $-\|R\|$ for 8 points, equally spaced in frequency. To facilitate comparison, the total width of the pattern, $\Delta \lambda^{2}$, has been scaled to match the width of the $\lambda^{2}$ sampling of Fig. 6. The righthand panel of Fig. 7 shows $\rho$ and $-\|R\|$ based on the same input data as Fig. 6. It is obvious that the RMTF of a 126 point sampling has much lower side lobes than an 8-point sampling. $n \pi$ ambiguities are completely eliminated.

The lefthand panel of Fig. 8 shows the same RMTF as the lefthand panel of Fig. 7. The two resonances to the left and right are due to the near-regularity of the sampling points in $\lambda^{2}$ space. If the frequency intervals at the lower frequencies are stretched more than at the intervals at higher frequencies, for example by making them decrease linearly with increasing frequency, one can make the pattern in $\lambda^{2}$ space less regular. The result is shown in the righthand panel of Fig. 8. The resonances are now lower and wider. If one requires the highest side lobes to be at least $5 \sigma$ lower than unity, then a total $S / N$ of 20 (7 per channel) is sufficient to prevent $n \pi$ ambiguities outside the main peak of the RMTF. Using the same requirement, in case of the 126 points, a $S / N$ of 6 in total ( 0.6 per channel) is enough to prevent $n \pi$ ambiguities outside the main peak of the RMTF.

$n \pi$ ambiguities are conceptually closely related to the grating response of a regularly spaced interferometer like the WSRT. When an interferometer only has baselines that are a multiple of some fixed distance, then its instantaneous synthesized beam is a collection of parallel fan beams. Each fan beam has the same peak amplitude. Therefore, without any further constraints it is impossible to determine in which fan beam the source is actually located. The same holds true for $\lambda^{2}$ sampling. If one only samples $\lambda^{2}$ space at regular intervals, there exist multiple solutions that fit the data equally well. These solutions correspond to peaks of unit amplitude in the RMTF: grating responses.

Figure 6 also shows that there are multiple minima of $\rho$ within the main lobe of the RMTF. These indicate uncertainties in the RM smaller than the width of the main peak. We shall now investigate the uncertainty in RM within the main peak of $\|R\|$.

The standard error in the RM when obtained by fitting a straight line to a plot of $\chi$ versus $\lambda^{2}$ is given by

$\sigma_{\mathrm{RM}}=\frac{\sigma_{\chi}}{\sigma_{\lambda^{2}} \sqrt{N-2}}$ 

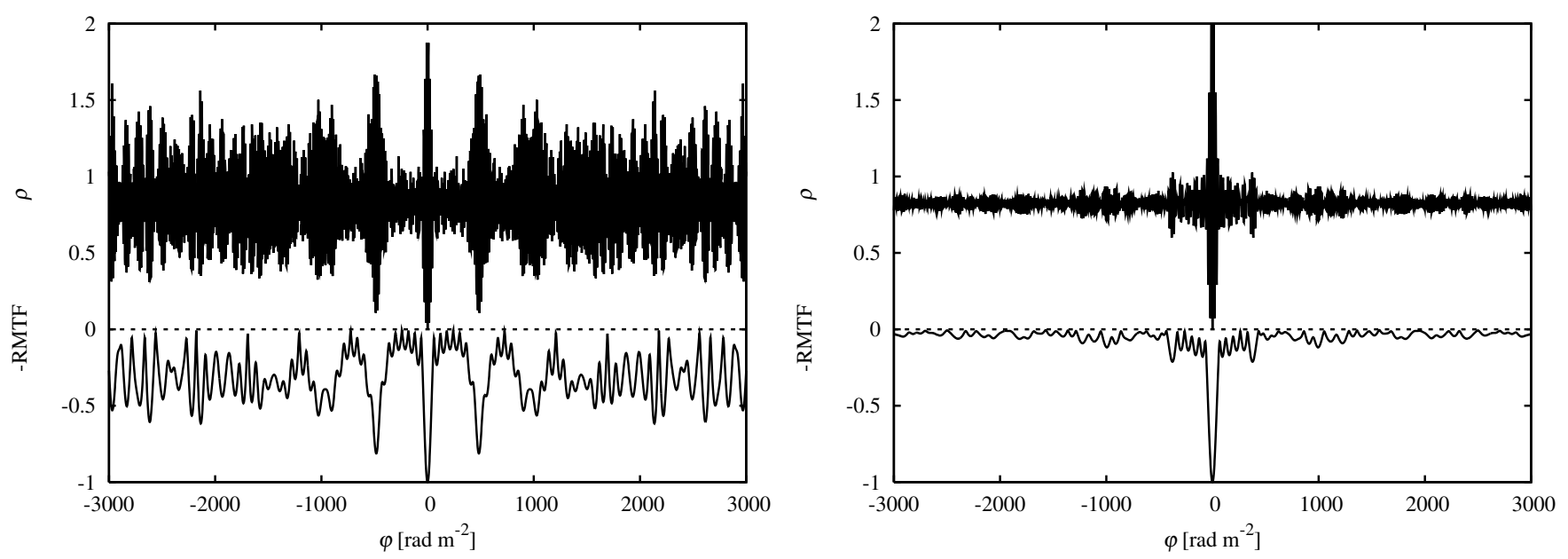

Fig. 7. Both plots show, from top to bottom, merit function $\rho$ and $-\|R(\phi)\|$. The lefthand panel uses eight sample points, equally spaced in frequency. The frequency sampling of the righthand panel is identical to the sampling used in Fig. 6 . The sampling in the lefthand panel has the same extent in $\lambda^{2}$-space as the sampling of the righthand panel.
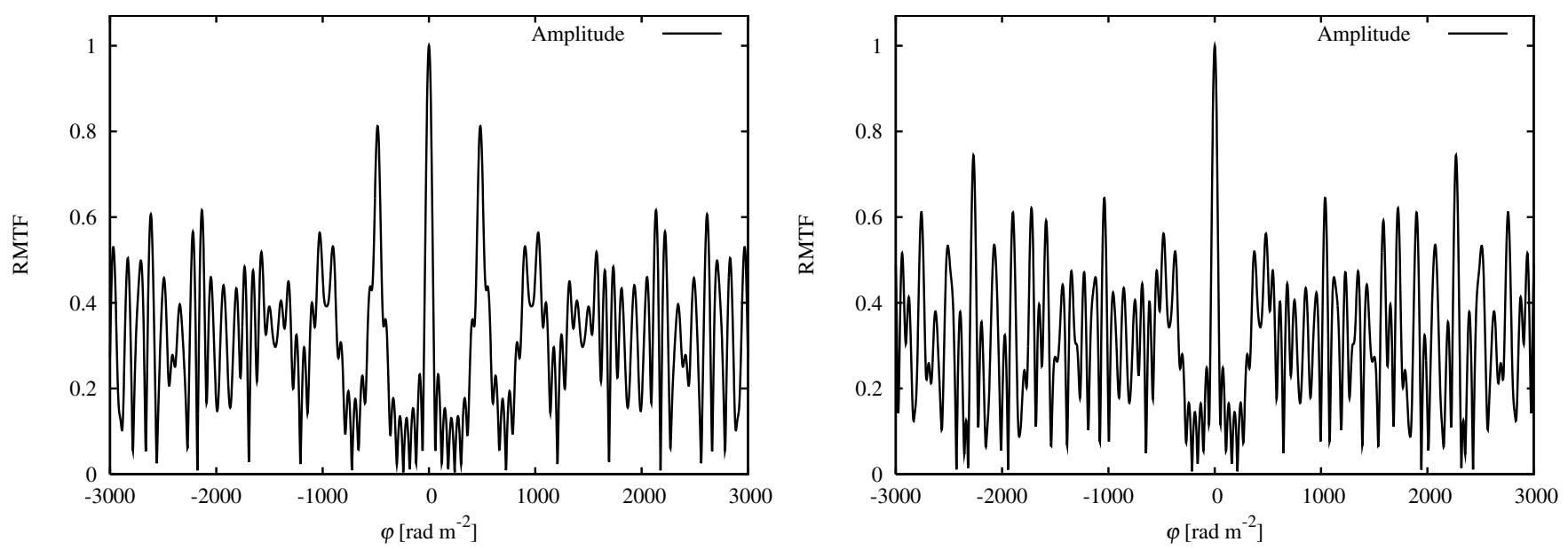

Fig. 8. This plot shows the effect of tweaking the exact frequencies of eight sampling points. The lefthand panel shows the same RMTF as the lefthand panel of Fig. 7. In the righthand plot, however, we stretched the frequency intervals such that low frequency intervals are wider than high frequency intervals. This eliminates the resonances from the lefthand plot.

$$
\sigma_{\lambda^{2}}=\sqrt{(N-1)^{-1} \sum_{i=1}^{N} \lambda_{i}^{4}-\lambda_{0}^{4}},
$$

where $\lambda_{0}$ is given by Eq. (32) and $N$ is the number of channels. $\sigma_{\chi}$ is either

$\sigma_{\chi}=\frac{1}{2} \frac{\sigma}{\|P\|}$

if $\sigma_{\mathrm{Q}} \approx \sigma_{\mathrm{U}}=\sigma$ or

$\sigma_{\chi}=\frac{\sqrt{U^{2} \sigma_{\mathrm{Q}}^{2}+Q^{2} \sigma_{\mathrm{U}}^{2}}}{2\|P\|^{2}}$

if $\sigma_{\mathrm{Q}}$ and $\sigma_{\mathrm{U}}$ differ by more than a factor of two or so. $\sigma_{\lambda^{2}}$ is the standard deviation of the distribution of $\lambda_{i}^{2}$ points. This is given by the frequency setup of the instrument. It is a measure of the effective width of the $\lambda^{2}$ distribution. Equation (52) is the result of straightforward error propagation. For reference, a full derivation is presented in Appendix A.
In RM-synthesis, one determines the RM of a single source along the line of sight by fitting, for example, a parabola to the main peak of $\|F(\phi)\|$. The detailed procedure is to first find the brightest point in a critically sampled Faraday dispersion function (2-3 points per $\delta \phi$ ), covering a wide range in $\phi$. This is followed by oversampling the region around the peak by a large factor. A parabolic fit to the 10-20 points directly surrounding the peak then yields the RM of the source.

We have simulated this procedure in order to get a quantitative idea of the typical error in RM that one obtains, given a certain noise level in the Stokes $Q$ and $U$ images, and a certain set of sample points $\lambda_{i}^{2}$. The results are shown in Fig. 9 . The total signal-to-noise ratio is equal to $\sqrt{N-2}\|P\| / \sigma$. The solid line is Eq. (52). The points are standard deviations in RM computed from 1000 iterations per $S / N$ ratio point. We have assumed the noise in Stokes $Q$ and $U$ to be equal and Gaussian. We see excellent agreement with the error expected for traditional $\lambda^{2}$ fitting (the straight line). At a $S / N$ ratio less than 4 , the points deviate strongly from the line. This is due to the fact that 


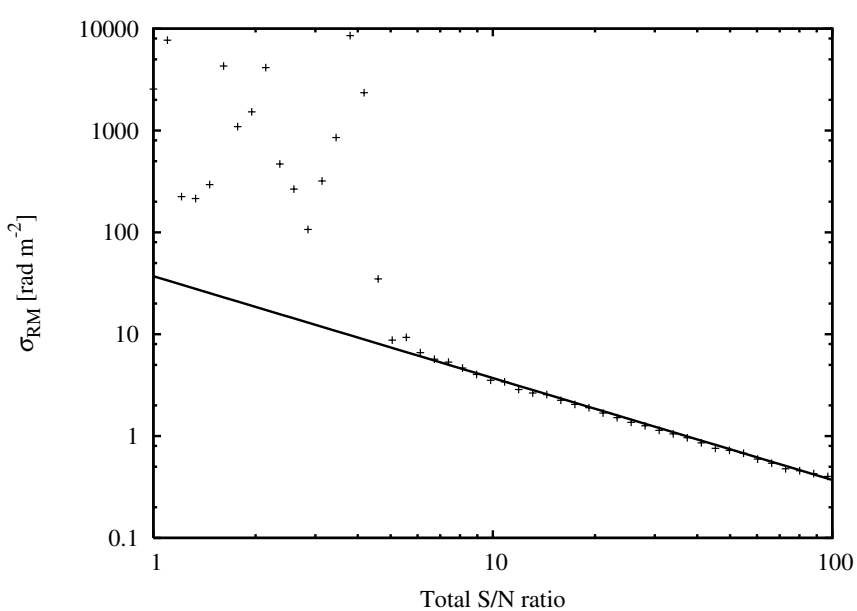

Fig. 9. Comparison between the standard error in RM obtained by traditional line fitting (line) to simulated RM-synthesis experiments where a parabola was fit to the main peak of the Faraday dispersion function (dots). The $126 \lambda^{2}$ points used for this figure are the same as the ones used in Fig. 3.

the non-Gaussianity of the noise in $P$ is only noticeable close to the origin of the complex plane. It is stressed that a total $S / N$ of 4 when having 126 channels implies a $S / N$ per channel of slightly less than 0.4 . It is impossible to determine a polarization angle with such a low $S / N$ in the case of standard $\lambda^{2}$ fitting.

\section{RM-synthesis with only $Q$ or $U$}

It is also possible to perform a RM-synthesis with Stokes $Q$ or $U$ only. There exist many radio observations that have produced only Stokes $I$ and $Q$, for example spectral line work with arrays equipped with linearly polarized feeds, or data from backends with limited correlator capacity. However, by using only one of the two Stokes parameters, one loses information about the sign of the Faraday depth.

The derivation is started with Eq. (25). The identities

$Q=\left(P+P^{*}\right) / 2$

$\mathrm{i} U=\left(P-P^{*}\right) / 2$

and

$G(x)=\int_{-\infty}^{\infty} g(t) \mathrm{e}^{a \mathrm{i} x t} \mathrm{~d} t \Leftrightarrow G^{*}(-x)=\int_{-\infty}^{\infty} g^{*}(t) \mathrm{e}^{a \mathrm{i} x t} \mathrm{~d} t$

are needed. After substituting Eqs. (56) and (57) into Eq. (25) and using Eq. (58), one obtains:

$$
\begin{aligned}
& \frac{1}{2}\left(\tilde{F}(\phi)+\tilde{F}^{*}(-\phi)\right)=K \int_{-\infty}^{+\infty} \tilde{Q}\left(\lambda^{2}\right) \mathrm{e}^{-2 \mathrm{i} \phi\left(\lambda^{2}-\lambda_{0}^{2}\right)} \mathrm{d} \lambda^{2} \\
& \frac{1}{2}\left(\tilde{F}(\phi)-\tilde{F}^{*}(-\phi)\right)=K \int_{-\infty}^{+\infty} \mathrm{i} \tilde{U}\left(\lambda^{2}\right) \mathrm{e}^{-2 \mathrm{i} \phi\left(\lambda^{2}-\lambda_{0}^{2}\right)} \mathrm{d} \lambda^{2} .
\end{aligned}
$$

The sensitivity to emission at low Faraday depth (less than one full rotation over the entire band) is limited by the orientation of the polarization vector. If the emission is mostly in $U$ at $\lambda=\lambda_{0}$, and hardly in $Q$, one will not retrieve the full total polarization when using Stokes $Q$ images only. On the other hand, if all emission is in $Q$, one apparently retrieves twice the actual total polarized intensity. Both cases are usually not very relevant, because RM-synthesis is mostly applied when emission at high Faraday depth is expected, where the polarization vector makes several turns over the observed band.

Figure 10 compares results of a complete RM-synthesis of data of the Perseus cluster, taken with the WSRT (de Bruyn $\&$ Brentjens 2005), to results of a $Q$-only RM-synthesis of the same dataset. It compares both the Galactic foreground emission at low Faraday depth, and the emission at higher Faraday depth that we attribute to the Perseus cluster. It is clearly seen that the noise in the $Q$-only images is increased with respect to the complete RM-synthesis. The bar-like feature at $\alpha \approx 3^{\mathrm{h}} 18^{\mathrm{m}}$, $\delta \approx+42^{\circ} 30^{\prime}$ is already visible in the $Q$-only images. This demonstrates that one actually can detect faint emission at high Faraday depths using only Stokes $Q$ or $U$ images. Unless the situation is simple, meaning only one discrete source along the line of sight, these images are unfortunately not useful in a quantitative sense. However, it is an efficient way to discover weak, Faraday rotated, polarized emission in existing datasets, which can then be followed up with full polarization observations.

\section{General experiment layout}

Three main parameters are involved when planning a rotationmeasure experiment, namely the channel width $\delta \lambda^{2}$, the width of the $\lambda^{2}$ distribution $\Delta \lambda^{2}$, and the shortest wavelength squared $\lambda_{\min }^{2}$. They are summarized in Fig. 11. These parameters determine respectively the maximum observable Faraday depth, the resolution in $\phi$ space, and the largest scale in $\phi$ space to which one is sensitive. Estimates for the FWHM of the main peak of the RMTF, the scale in $\phi$ space to which sensitivity has dropped to $50 \%$ and the maximum Faraday depth to which one has more than $50 \%$ sensitivity are approximately

$\delta \phi \approx \frac{2 \sqrt{3}}{\Delta \lambda^{2}}$

$\max$-scale $\approx \frac{\pi}{\lambda_{\min }^{2}}$

$\left\|\phi_{\max }\right\| \approx \frac{\sqrt{3}}{\delta \lambda^{2}}$.

In these equations we assumed a top hat weight function which is 1 between $\lambda_{\min }^{2}$ and $\lambda_{\max }^{2}$ and zero elsewhere.

It is interesting to compare Eqs. (61) and (62). This is where the analogy between RM-synthesis and regular synthesis imaging breaks down. In synthesis imaging, the width of the synthesized beam is inversely proportional to the maximum absolute $u v$ vector. That is, the distance between the origin and the $u v$ point most distant from it. The maximum scale that one can measure depends on the shortest baseline. Therefore one is always maximally sensitive to structures smaller than the width of the synthesized beam.

This is quite different in RM-synthesis. In RM-synthesis it is possible that a source is unresolved in the sense that its extent in $\phi$ is less than the width of the RMTF, yet "resolved" out because one has not sampled the typical $\phi$-scale of the source due to lack of small $\lambda^{2}$ points. Equation (61) shows that the 
Q $\mathrm{Q}+\mathrm{U}$

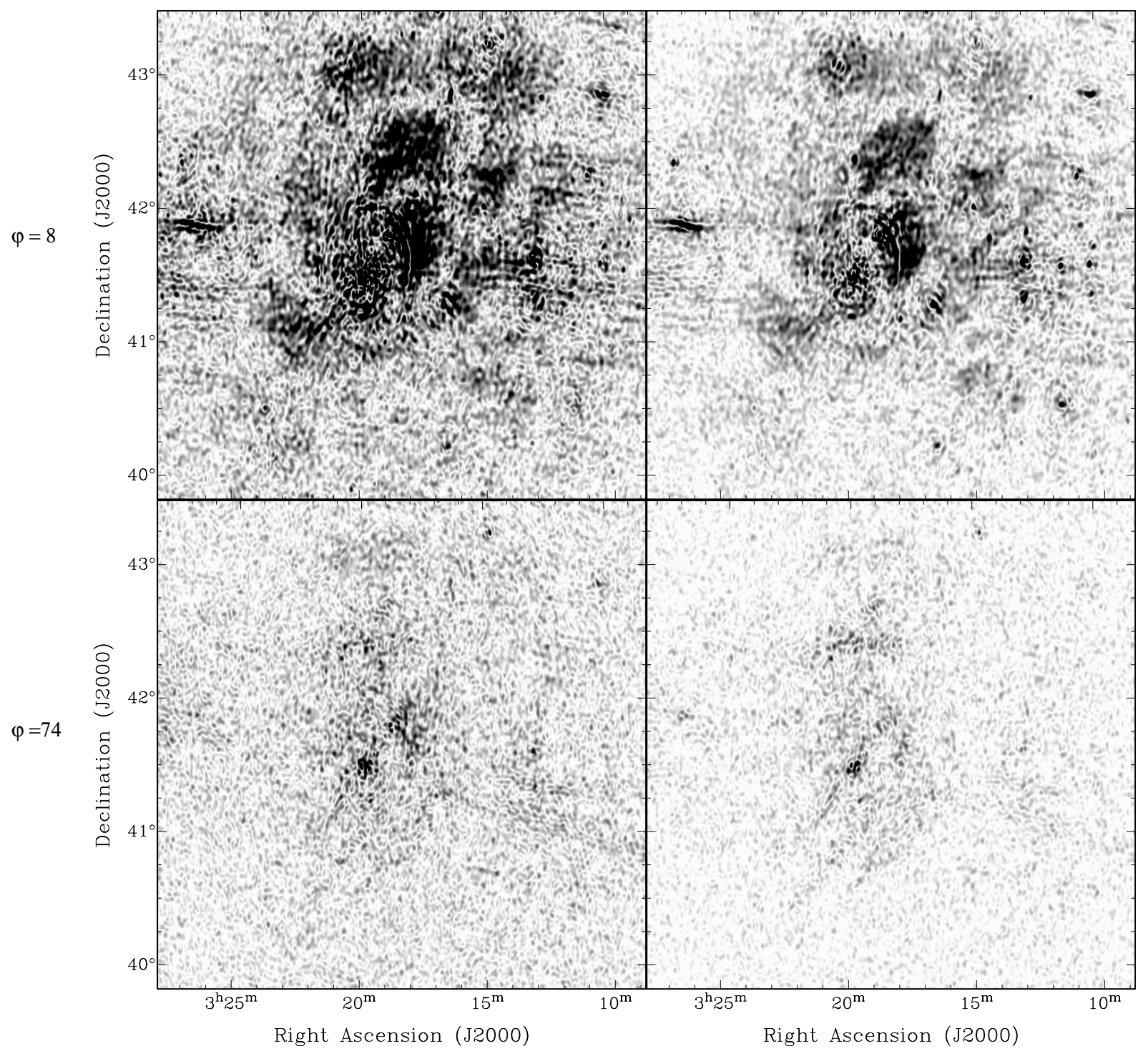

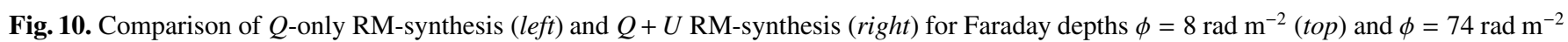
(bottom). The panels show total linear polarization in grey scale. The scale runs from $0.15 \mathrm{mJy}$ beam $^{-1} \mathrm{rmtf}^{-1}$ to $1.5 \mathrm{mJy} \mathrm{beam}^{-1} \mathrm{rmtf}^{-1}$.

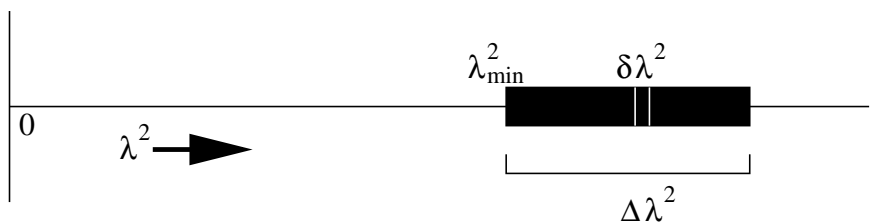

Fig. 11. The three instrumental parameters that determine the output of a Faraday rotation experiment.

width of the RMTF depends on the width of the $\lambda^{2}$ distribution, not on the largest $\lambda^{2}$ measured. Nevertheless the largest scale in $\phi$ that one is sensitive to is set by the smallest $\lambda^{2}$ as is shown in Eq. (62). In order to truly resolve Faraday thick clouds in $\phi$ space in the sense that one could see internal structure, the main peak of the RMTF should be narrower than the maximum scale to which one is sensitive. Because $2 \sqrt{3} \approx \pi$, the requirement for resolving Faraday thick structures is

$\lambda_{\min }^{2}<\Delta \lambda^{2}$.

See Appendix B for simulations illustrating this point.

For deconvolution the RMTF should be known as accurately as possible for all sources within the field of view and along the line of sight. The main problems are:

- frequency dependence of the primary beam attenuation;

- frequency dependence of the instrumental polarization;

- the intrinsic emission spectra of the sources;

- frequency dependence of the synthesized beam size.

The last point is easily compensated for by using the same $u v$ taper for all channel maps. The first two points can be alleviated to a large extent by observing in mosaic mode. The primary beam attenuation can cause very steep artificial spectral 
indices. These can be as steep as $\alpha \approx-1.5$ near half power and $\alpha \approx-5$ near the $-10 \mathrm{~dB}$ point at the WSRT. Fortunately, the primary beam attenuation is usually known accurately. Therefore one can predict $W\left(\lambda^{2}\right)$ for any point in the field of view, enabling one to accurately compute the local RMTF at any pixel using for example Eq. (37).

After primary beam correction, one should align the channel maps spectrally. Our preferred method is to determine the average total intensity of a large sample of sources, and scale the images until the average of the ensemble in a particular channel map matches the value at $\lambda=\lambda_{0}$. Using this approach, the spectra of as yet undetected emission should be approximately flat to within a spectral index range of \pm 1 . Of course one could flatten source spectra on an individual basis. This is only useful if one is interested in bright sources that are easily detected in individual channels.

A convenient property of RM-synthesis is that more-or-less frequency independent instrumental problems end up at $\phi=0$, convolved with the RMTF. This means that instrumental problems are highly reduced at higher absolute Faraday depths. In other words: at high Faraday depth, we "wind-up" the instrumental polarization problems, while "unwinding" the Faraday rotated cosmic polarization signals.

\section{Conclusions}

We have extended the work of Burn (1966) to the cases of limited sampling of $\lambda^{2}$ space and some spectral dependencies. We have introduced the RMTF, which is an excellent predictor of $n \pi$ ambiguity problems in the frequency setup. RM-synthesis can be implemented very efficiently on modern computers. For example, a RM- synthesis of 126 input maps of $1024^{2}$ pixels, yielding $3 \times 100$ output maps of $1024^{2}$ pixels $(P, Q$, and $U$ ) takes less than $5 \mathrm{~min}$ on a laptop equipped with a $2 \mathrm{GHz}$ Intel Pentium processor and $512 \mathrm{MB}$ of RAM.

Because the analysis is easily applied to wide fields, one can conduct very fast RM surveys of weak sources. Difficult situations, for example multiple sources along the line of sight, are easily detected. Under certain conditions, it is even possible to recover the emission as a function of Faraday depth within a single cloud of ionized gas.

Instrumental problems that are weakly frequency independent, or have a very characteristic frequency dependence, are easily separated from cosmic signals that are only subject to Faraday rotation.
Rotation measure synthesis has already been successful in discovering widespread, weak, polarized emission associated with the Perseus cluster (de Bruyn \& Brentjens 2005). In simple, high signal to noise situations it is as good as traditional linear fits to $\chi$ versus $\lambda^{2}$ plots. However, when the situation is more complex, or very weak polarized emission at high rotation measures is expected, it is the only viable option.

Acknowledgements. We acknowledge Robert Braun, Torsten Enßlin and Peter Katgert for useful and vivid discussions on the subject. The Westerbork Synthesis Radio Telescope is operated by ASTRON (Netherlands Foundation for Research in Astronomy) with support from the Netherlands Foundation for Scientific Research (NWO).

\section{References}

Burn, B. J. 1966, MNRAS, 133, 67

Conway, R. G., Kellermann, K. I., \& Long, R. J. 1963, MNRAS, 125, 261

Conway, R. G., \& Strom, R. G. 1985, A\&A, 146, 392

de Bruyn, A. G. 1996, RM-synthesis via wide-band low-frequency polarimetry, Tech. Rep. NFRA Note 655, ASTRON, Dwingeloo

de Bruyn, A. G., \& Brentjens, M. A. 2005, A\&A, 441, 931

Dolag, K., Vogt, C., \& Enßlin, T. A. 2004

[arXiv: astro-ph/0401337]

Gardner, F. F. \& Whiteoak, J. B. 1966, ARA\&A, 4, 245

Kronberg, P. P. 1994, Rep. Prog. Phys., 57, 325

Le Roux, E. 1961, Annales d'Astrophysique, 24, 71

Mitra, D., Wielebinski, R., Kramer, M., \& Jessner, A. 2003, A\&A, 398, 993

Sokoloff, D. D., Bykov, A. A., Shukurov, A., et al. 1998, MNRAS, 299, 189

Sokoloff, D. D., Bykov, A. A., Shukurov, A., et al. 1999, MNRAS, 303, 207

Squires, G. L. 2001, Practical Physics (Cambridge University Press)

Strom, R. G., \& Conway, R. G. 1985, A\&AS, 61, 547

Vallee, J. P. 1980, A\&A, 86, 251

Vallee, J. P. 1997, Fundamentals of Cosmic Physics, 19, 1

Vogt, C., Dolag, K., \& Enßlin, T. A. 2004

[arXiv: astro-ph/0401216]

Weisberg, J. M., Cordes, J. M., Kuan, B., et al. 2004, ApJS, 150, 317

Westfold, K. C. 1959, ApJ, 130, 241

Widrow, L. M. 2002, Rev. Mod. Phys., 74, 775

Woltjer, L. 1958, Bull. Astron. Inst. Netherlands, 14, 39 


\section{Online Material}




\section{Appendix A: Standard errors in RM estimations}

The expected standard errors in RM/Faraday depth and $\chi_{0}$ are useful quantities when planning a rotation measure experiment. In this appendix we present a formal derivation.

From Eqs. (7) and (8) we have

$$
\begin{aligned}
& \|P\|=\sqrt{Q^{2}+U^{2}} \\
& \chi=\frac{1}{2} \tan ^{-1} \frac{U}{Q} .
\end{aligned}
$$

We discriminate two cases. The first is $\sigma_{\mathrm{Q}} \approx \sigma_{\mathrm{U}}=\sigma$, the second is $\sigma_{\mathrm{Q}} \gg \sigma_{\mathrm{U}}$ or $\sigma_{\mathrm{Q}} \ll \sigma_{\mathrm{U}}$, where $\gg$ and $\ll$ indicate a difference of more than a factor of two. $\sigma_{\mathrm{Q}}$ and $\sigma_{\mathrm{U}}$ are the RMS image noise in individual $Q$ and $U$ channel maps.

The derivation is done in two steps. First we derive the standard error in the polarization angle and total polarization, $\sigma_{\chi}$ and $\sigma_{\mathrm{P}}$ of measurements in individual channels. Then we apply standard results for the least squares fit of a straight line to obtain $\sigma_{\phi}$ and $\sigma_{\chi 0}$, the standard errors in rotation measure / Faraday depth and the polarization angle at $\lambda=0$.

Error propagation (Squires 2001) gives us

$$
\begin{aligned}
& \sigma_{\mathrm{P}}^{2}=\left(\frac{\partial\|P\|}{\partial Q}\right)^{2} \sigma_{\mathrm{Q}}^{2}+\left(\frac{\partial\|P\|}{\partial U}\right)^{2} \sigma_{\mathrm{U}}^{2} \\
& \sigma_{\chi}^{2}=\left(\frac{\partial \chi}{\partial Q}\right)^{2} \sigma_{\mathrm{Q}}^{2}+\left(\frac{\partial \chi}{\partial U}\right)^{2} \sigma_{\mathrm{U}}^{2} .
\end{aligned}
$$

The partial derivatives for $\sigma_{\mathrm{P}}$ are

$$
\begin{aligned}
& \left(\frac{\partial\|P\|}{\partial Q}\right)^{2}=\frac{Q^{2}}{Q^{2}+U^{2}} \\
& \left(\frac{\partial\|P\|}{\partial U}\right)^{2}=\frac{U^{2}}{Q^{2}+U^{2}} .
\end{aligned}
$$

Inserting this in Eq. (A.3) gives

$\sigma_{\mathrm{P}}^{2}=\frac{Q^{2}}{\|P\|^{2}} \sigma_{\mathrm{Q}}^{2}+\frac{U^{2}}{\|P\|^{2}} \sigma_{\mathrm{U}}^{2}$.

In the most general case, this cannot be simplified any further. Nevertheless, when $\sigma_{\mathrm{Q}} \approx \sigma_{\mathrm{U}}=\sigma$, Eq. (A.7) can be simplified to

$\sigma_{\mathrm{P}}^{2}=\sigma^{2}$

The partial derivatives needed for $\sigma_{\chi}^{2}$ are

$$
\begin{aligned}
& \frac{\partial \frac{1}{2} \tan ^{-1} \frac{U}{Q}}{\partial Q}=\frac{1}{4} \frac{U^{2}}{\left(Q^{2}+U^{2}\right)^{2}} \\
& \frac{\partial \frac{1}{2} \tan ^{-1} \frac{U}{Q}}{\partial U}=\frac{1}{4} \frac{Q^{2}}{\left(Q^{2}+U^{2}\right)^{2}} .
\end{aligned}
$$

Inserting these in Eq. (A.4) gives

$$
\sigma_{\chi}^{2}=\frac{U^{2} \sigma_{\mathrm{Q}}^{2}+Q^{2} \sigma_{\mathrm{U}}^{2}}{4\|P\|^{4}} .
$$

This result can only be simplified further if $\sigma_{\mathrm{Q}} \approx \sigma_{\mathrm{U}}=\sigma$ :

$$
\sigma_{\chi}^{2}=\frac{1}{4} \frac{\sigma^{2}}{\|P\|^{2}}
$$

When fitting a straight line $y=a x+b$ to data with equal estimated standard errors per data point, the standard error in the slope of the line is (Squires 2001)

$\sigma_{\mathrm{a}}^{2} \approx \frac{1}{N-2} \frac{\Sigma_{i}\left(y_{i}-a x_{i}-b\right)^{2}}{\Sigma_{i} x_{i}^{2}-N^{-1}\left(\Sigma_{i} x_{i}\right)^{2}}$.

Now one substitutes $\phi=a, \chi_{0}=b, \chi=y$, and $\lambda^{2}=x$. A rotation measure of 0 may be assumed without loss of generality. The equation then becomes

$\sigma_{\phi}^{2}=\frac{1}{N-2} \frac{\Sigma_{i} \chi_{i}^{2}}{\Sigma_{i}\left(\lambda_{i}^{2}\right)^{2}-N^{-1}\left(\Sigma_{i} \lambda_{i}^{2}\right)}$.

The variance of the polarization angle distribution is given by

$\sigma_{\chi}^{2}=\frac{1}{N-1} \Sigma_{i} \chi_{i}^{2}-\langle\chi\rangle$

where \langle\rangle denotes averaging. Combining Eqs. (A.14) and (A.15) and using $\langle\chi\rangle=0$ one obtains

$\sigma_{\phi}^{2}=\frac{N-1}{N-2} \frac{\sigma_{\chi}^{2}}{\Sigma\left(\lambda_{i}^{2}\right)^{2}-N^{-1}\left(\Sigma_{i} \lambda_{i}^{2}\right)^{2}}$.

The variance of the $\lambda^{2}$ distribution is

$\sigma_{\lambda^{2}}^{2}=\frac{1}{N-1}\left(\Sigma_{i} \lambda_{i}^{4}-N^{-1}\left(\Sigma_{i} \lambda_{i}^{2}\right)^{2}\right)$.

This is a measure of the effective width of the $\lambda^{2}$ coverage.

Equation (A.16) may now be simplified by substituting Eqs. (A.17) and (A.12). The final result is:

$\sigma_{\phi}^{2}=\frac{\sigma^{2}}{4(N-2)\|P\|^{2} \sigma_{\lambda^{2}}^{2}}$

The standard error in in $\chi_{0}, \sigma_{\chi_{0}}$ is derived in a similar fashion. One starts with

$\sigma_{\mathrm{b}}^{2} \approx\left(\frac{\Sigma_{i} x_{i}^{2}-N^{-1}\left(\Sigma x_{i}\right)^{2}}{N}+\langle x\rangle^{2}\right) \sigma_{\mathrm{a}}^{2}$.

After substituting Eqs. (A.17) and (32) and some rearranging, the final result is:

$\sigma_{\chi_{0}}^{2}=\frac{\sigma^{2}}{4(N-2)\|P\|^{2}}\left(\frac{N-1}{N}+\frac{\lambda_{0}^{4}}{\sigma_{\lambda^{2}}^{2}}\right)$.

\section{Appendix B: Example simulations}

In this appendix we show, as an illustration, three model runs of an RM-synthesis of an artificial Faraday dispersion function, measured with a realistic frequency sampling. We hope that these figures aid in understanding the most important aspects of RM-synthesis specifically and rotation measure work in general.

Sources that are extended in the plane of the sky have their surface brightness measured in Jy per steradian. For point sources the flux in Jy is sufficient to characterize it. The respective brightness units for sources that are both extended in the plane of the sky and in Faraday depth are Jy steradian ${ }^{-1}$ 
M. A. Brentjens and A. G. de Bruyn: RM-synthesis, Online Material p 3
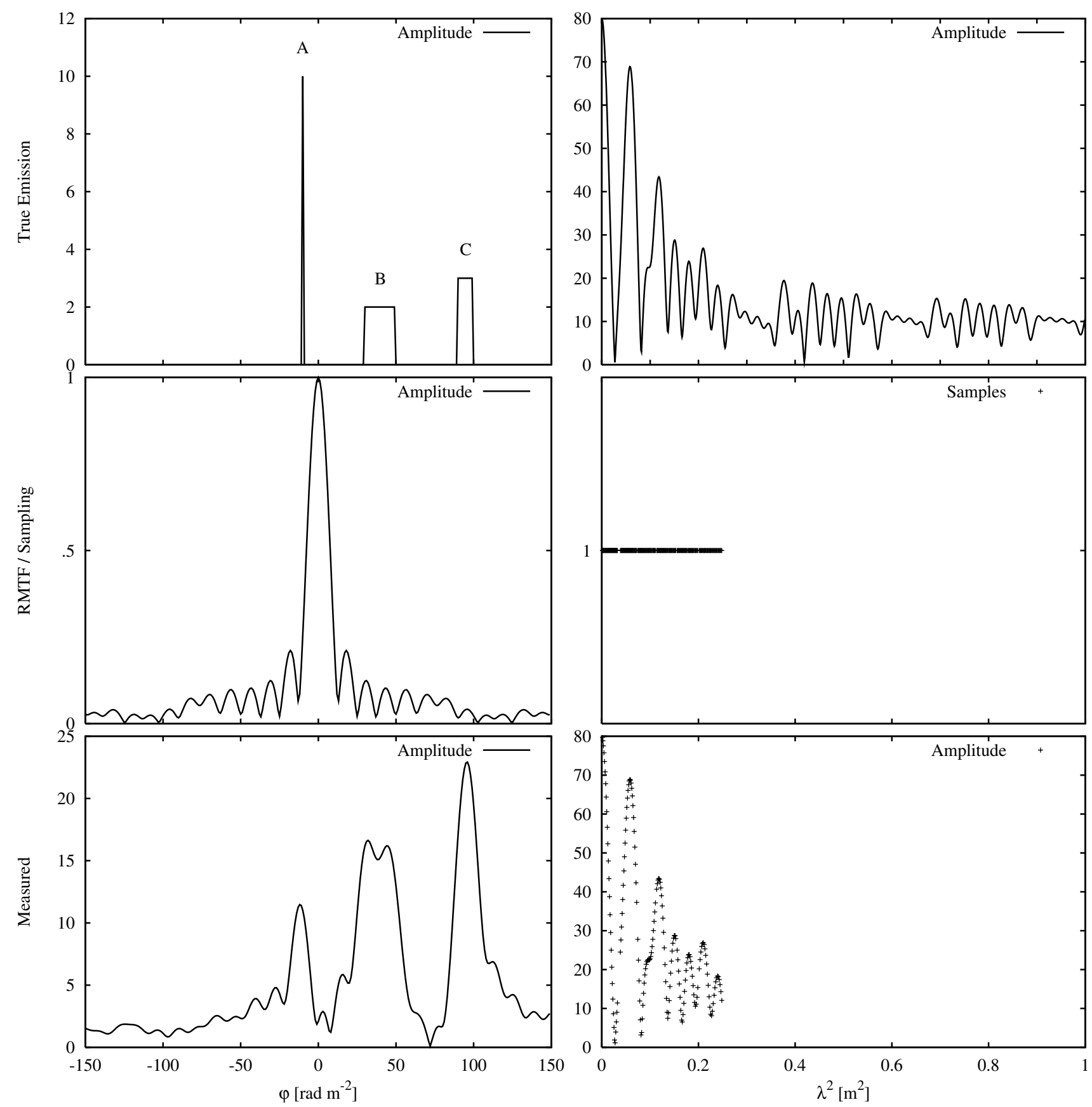

Fig. B.1. Wavelength range: $3.6-50 \mathrm{~cm}$.

$\left.(\operatorname{rad~m})^{-2}\right)^{-1}$ or Jy m $\mathrm{m}^{2} \mathrm{rad}^{-3}$. Sources that are extended in the plane of the sky and point-like in $\phi$ space have their brightness in $\phi$ space measured in Jy steradian ${ }^{-1}$. The brightness of the measured Faraday dispersion function has units of Jy (beam on the sky $)^{-1}(\mathrm{rmtf})^{-1}$. Sources that are point-like in the plane of the sky have the steradian ${ }^{-1}$ or (beam on the sky) $)^{-1}$ removed.

In order to keep the units simple, we made all simulated sources point-like in the sky plane. Hence the units used in the figures in this appendix are:

- top right and bottom right: Jy;

- middle left and middle right: dimensionless;

- top left: Jy $\mathrm{m}^{2} \mathrm{rad}^{-1}$;

- bottom left: Jy $\mathrm{rmtf}^{-1}$.
The lefthand column of Fig. B.1, Fig. B.2, and Fig. B.3 is the situation in $\phi$ space. The righthand column is the corresponding situation in $\lambda^{2}$ space. Pictures in one row are converted into each other by Fourier relations (15) (left to right) and (25) (right to left). The top row is the input situation. It is the same in all three figures. The lefthand panel represents the polarized flux per unit Faraday depth in Jy per $\left(\mathrm{rad} \mathrm{m}^{-2}\right)$. The righthand panel is the polarized flux in Jy. The example sources all have flat spectra. The second row shows the RMTF in the lefthand panel and the sampling function in the righthand panel. The third row shows the result of applying the second row to the first row. That is, multiplication of top right and middle right give bottom right and top left convolved with middle left after 
M. A. Brentjens and A. G. de Bruyn: RM-synthesis, Online Material p 4
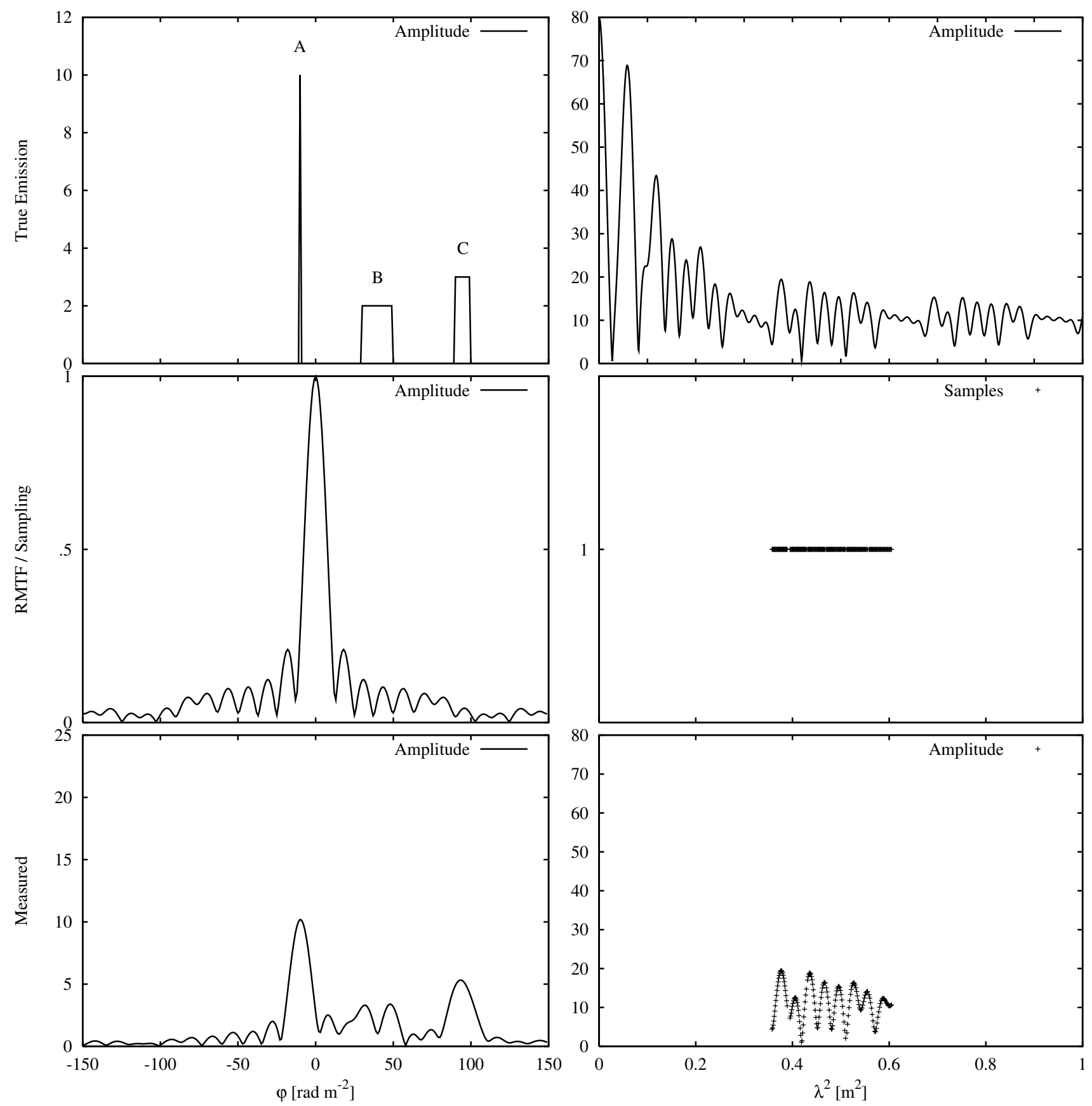

Fig. B.2. Wavelength range: $60-78 \mathrm{~cm}$.

$\lambda^{2}$ bandpass filtering gives bottom left. Except for the weight of the samples in $\lambda^{2}$ space, all quantities are complex. For clarity, we have only shown the amplitude here.

The RMTF in all three figures is the same because the pattern and width of the $\lambda^{2}$ coverage is exactly the same for all of them. The only difference is the absolute position of the pattern. Figure B.1 has $\lambda_{\min }^{2}=3.6^{2} \mathrm{~cm}^{2}$, Fig. B. 2 has $\lambda_{\min }^{2}=60^{2} \mathrm{~cm}^{2}$, and Fig. B. 3 has $\lambda_{\min }^{2}=81^{2} \mathrm{~cm}^{2}$.

The three sources in this simulation have different properties to illustrate different cases.

A: $\phi=-10 \mathrm{rad} \mathrm{m}^{-2}$, delta function of $\phi$, total flux density is $10 \mathrm{Jy}$. In synthesis imaging, this would be the equivalent of a point source;
B: $30 \leq \phi \leq 50 \mathrm{rad} \mathrm{m}^{-2}$, multiple RMTFs wide in $\phi, F(\phi)=$ $2 \mathrm{Jy} \mathrm{m}^{2} \mathrm{rad}^{-1}$, total flux density is $40 \mathrm{Jy}$. In synthesis imaging, this would be the equivalent of an extended source;

C: $90 \leq \phi \leq 100 \mathrm{rad} \mathrm{m}^{-2}$, roughly one RMTF wide in $\phi$, $F(\phi)=3 \mathrm{Jy} \mathrm{m}^{2} \mathrm{rad}^{-1}$, total flux density is $30 \mathrm{Jy}$. In synthesis imaging, this would be the equivalent of a barely resolved source.

Because source A is a delta function with respect to $\phi$, the amplitude of its Fourier transform is the same at all $\lambda^{2}$. Therefore, the source appears in all three figures with equal amplitude. The peak is slightly higher than $10 \mathrm{Jy} \mathrm{rmtf}^{-1}$ in Fig. B.1 and Fig. B.2 due to side lobes of the responses of sources B and C. In Fig. B.3 the retrieved flux of sources B and C has collapsed 
M. A. Brentjens and A. G. de Bruyn: RM-synthesis, Online Material p 5
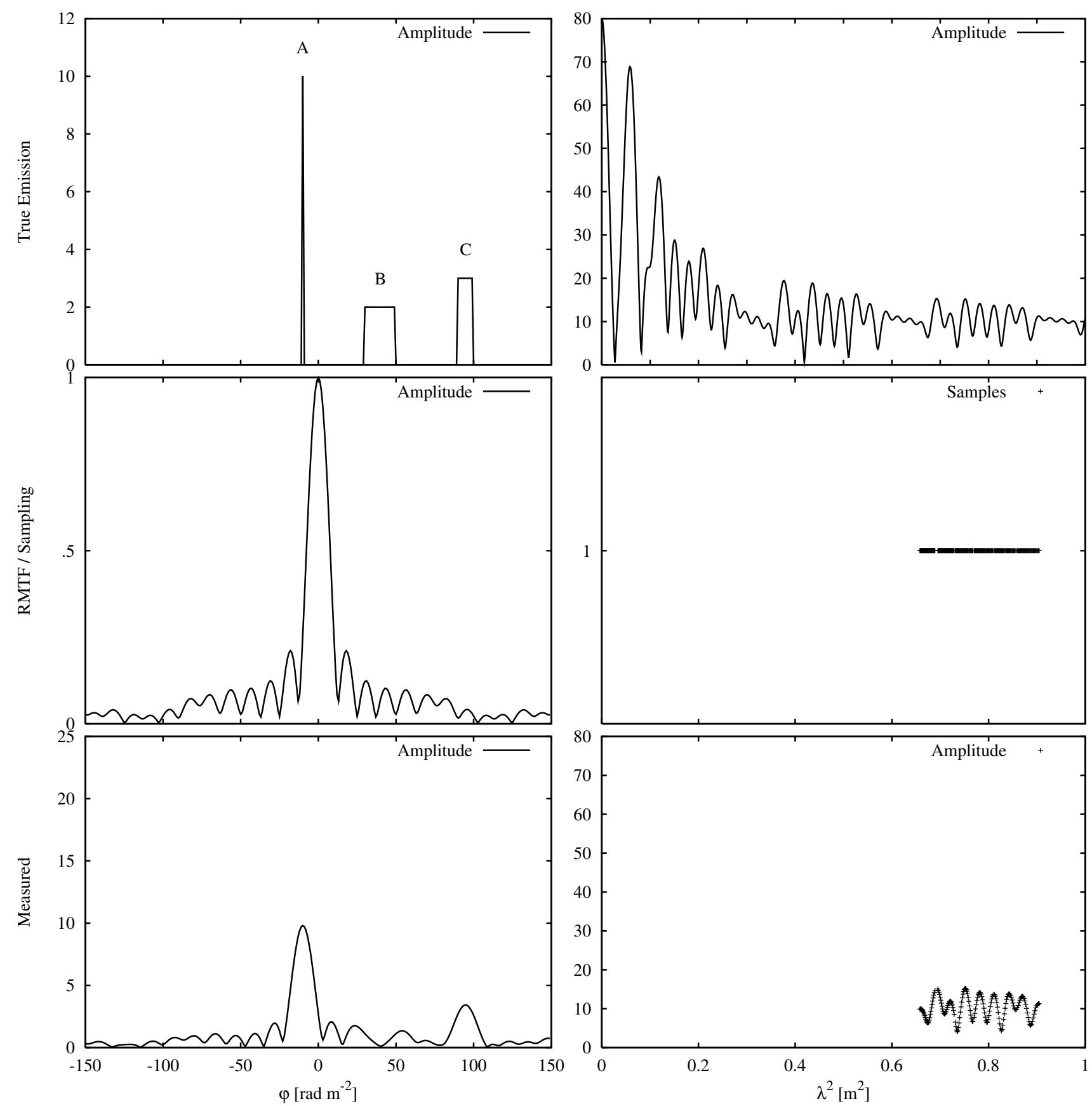

Fig. B.3. Wavelength range: $81-95 \mathrm{~cm}$.

so much that the response to source A is practically the same as the RMTF, especially to the left of source A.

Source B represents the other extreme. Being several RMTFs wide, one requires $\lambda_{\text {min }}^{2} \ll \Delta \lambda^{2}$ in order to recover the full flux of the source. Only Fig. B.1 meets this requirement. In Fig. B.2, only two bumps at the edges of the source remain. Because in Fig. B. 2 we only sample smaller scales in $\phi$ due to the larger $\lambda_{\min }^{2}$, the only parts of source $\mathrm{B}$ that remain are the parts where these smaller scales are important: the edges. Source B has practically disappeared in Fig. B.3.

Source $\mathrm{C}$ is of an intermediate type. Because its typical $\phi$ scale is narrower than source $\mathrm{B}$, there is a larger fraction of the total flux recovered in Figs. B.2 and B.3.

In analogy to radio interferometric observations, one could state that the $\lambda^{2}$ sampling in Fig. B.1 corresponds to a connected element array, where one samples all scales up to $\lambda_{\max }^{2}$ approximately equally well. Figure B.3 corresponds to a VLBI observation, where one misses the short spacings and therefore is insensitive to extended emission. A fundamental difference with radio interferometry is that the resolution in $\phi$ space is determined by the width of the $\lambda^{2}$ distribution, $\Delta \lambda^{2}$, and not by the largest $\lambda^{2}$ sampled. Hence one could encounter situations where a source is not resolved in the sense that the thickness of the source in $\phi$ is much less than the width of the RMTF, while at the same time it $i s$ resolved out in the sense that one has not sampled sufficiently short $\lambda^{2}$ points to detect the source. 